\title{
Revisiting helicity parton distributions at a future electron-ion collider
}

\author{
Ignacio Borsa ${ }^{*},{ }^{*}$ Gonzalo Lucero, ${ }^{\dagger}$ and Rodolfo Sassot $\odot^{*}$ \\ Departamento de Física and IFIBA, Facultad de Ciencias Exactas y Naturales, \\ Universidad de Buenos Aires, Ciudad Universitaria, \\ Pabellón 1 (1428) Buenos Aires, Argentina \\ Elke C. Aschenauer $\circledast^{\S}$ and Ana S. Nunes® \\ Physics Department, Brookhaven National Laboratory, Upton, New York 11973, USA \\ (Received 19 July 2020; accepted 14 October 2020; published 23 November 2020)
}

\begin{abstract}
We studied the impact of future electron ion collider inclusive and semi-inclusive polarized deep inelastic scattering data will have on the determination of the helicity parton distributions. Supplementing the Monte Carlo sampling variant of the DSSV14 analysis with pseudodata on polarized inclusive and semi-inclusive electron-proton deep inelastic scattering with updated uncertainty estimates and for two different center-of-mass-system energies, $\sqrt{s}=44.7 \mathrm{GeV}$ and $\sqrt{s}=141.4 \mathrm{GeV}$, respectively, and on inclusive electron-helium collisions at $\sqrt{s}=115.2 \mathrm{GeV}$, we find a remarkable improvement in the determination of the helicity distributions, especially at low parton momentum fraction $x$. While inclusive electron-proton data at the lowest energy configuration constrain significantly the gluon polarization down to $x \sim 10^{-4}$, the higher energy configuration strengthens the constraint and extends it one decade further. On the other hand, semi-inclusive data achieves the hitherto elusive flavor separation for sea quarks that cannot be obtained from any other inclusive electromagnetic measurement. Collisions with helium complement inclusive proton measurements, pushing the constraints on the combined quark plus antiquark $u, d$ and $s$ polarizations to an unprecedented level.
\end{abstract}

DOI: $10.1103 /$ PhysRevD.102.094018

\section{INTRODUCTION AND MOTIVATION}

Ever since the pioneering measurements of the EMC experiment at CERN suggested that quarks and antiquarks are only responsible for a small fraction of the proton spin [1], thus challenging the naive quark-parton model picture, the way in which the proton spin builds up from its fundamental constituents, quarks and gluons, has remained an open question [2].

While the amount of spin carried by quarks and antiquarks has been confirmed to be much less than the expected $\frac{\hbar}{2}$ value by subsequent measurements of polarized deep inelastic scattering (DIS) of leptons off fixed proton, deuteron, and helium targets at SLAC, CERN, Deutsches Elektronen-Synchrotron (DESY) and JLAB [2], it is still

\footnotetext{
*iborsa@df.uba.ar

†glucero@df.uba.ar

sassot@df.uba.ar

§elke@bnl.gov

anunes@bnl.gov
}

Published by the American Physical Society under the terms of the Creative Commons Attribution 4.0 International license. Further distribution of this work must maintain attribution to the author(s) and the published article's title, journal citation, and DOI. Funded by SCOAP ${ }^{3}$. unclear how much of the missing spin is carried by the gluons and how much should be associated to the orbital angular momentum of partons.

In this quest for the origin of the proton spin, the measurement of hadrons and jets produced at high transverse momentum in polarized proton-proton collisions at the Brookhaven National Laboratory-Relativistic Heavy Ion Collider (BNL-RHIC) has also set a crucial milestone [3]. Since these measurements receive their most significant contributions from gluon initiated processes, the RHIC spin program has provided a fundamental grip on the gluon polarization, showing a non-negligible contribution to the spin of the proton, albeit in a restricted gluon momentum fraction region $[4,5]$. In spite of the very successful RHIC spin program, the gluon helicity distribution can be at best conjectured for values of the momentum fraction below $x \sim 10^{-2}$.

Besides these fundamental questions on the role of the gluon polarization, and that of the orbital angular momentum, the way in which each particular quark flavor contributes to the proton spin is also work in progress. In the naive quark model there is a fundamental relation between quark spin and flavor. However, we still lack a clear picture of how quarks of radiative origin modify at short distances the quark-parton model paradigm. 
First determinations of flavor discriminated helicity parton densities were based solely on rather simple symmetry assumptions. Even though these were later replaced or supplemented with semi-inclusive deep inelastic scattering (SIDIS) data, and recently with data from charged weak vector boson production in proton-proton collisions, the kinematical coverage for this data is limited and, therefore, nonconclusive [2].

The Electron Ion Collider (EIC) is designed to be the most powerful tool to answer the above mentioned questions, quantifying the way in which gluons and quarks of different flavors make up for the total spin of the proton [6]. Specifically, with unprecedented precision and a wide coverage in the parton momentum fraction $x$, and in the photon virtuality $Q^{2}$, the EIC is expected to provide new insights into the gluon polarization. In particular, extremely precise measurements of the polarized structure function $g_{1}$ and its scaling violations for values of $x$ two decades below the limit of $10^{-2}$ will lead to stringent constraints on the helicity distributions, absent in current global analysis. On the other hand, a very thorough program of SIDIS measurements (polarized and unpolarized) at the EIC will allow to develop a much more precise picture of the relations between spin and flavor, the role of sea quarks in the proton polarization, and the degree of flavor and charge symmetry breaking. It has already been shown that SIDIS measurements are instrumental for discriminating between quark and antiquarks but will be extended one step further in precision and range at an EIC. Although, naively, one would expect quarks to become charge and flavor symmetric, as well as unpolarized at small enough parton momentum fraction, none of the available estimates predict where exactly QCD radiative mechanisms overcome nonperturbative effects. We note that unpolarized measurements of the SIDIS cross-sections in the same broad kinematic region, where the spin dependent data is obtained, are expected to allow us to further refine the current perturbative description of SIDIS processes, namely providing fragmentation functions of unprecedented precision [7]. Additionally, even though inclusive electronhelium collision cannot disentangle quarks from antiquarks, very precise measurements of this type complement inclusive and semi-inclusive electron-proton data by providing access to the total (quark plus antiquark) polarization for $\mathrm{u}, \mathrm{d}$ and $\mathrm{s}$ quarks with extreme precision down to very small parton momentum fractions.

In order to asses the impact of future EIC data in the determination of the helicity parton distributions, we have to perform a next-to-leading order (NLO) global analysis adding to the DSSV14 dataset [4], inclusive and semiinclusive polarized DIS pseudodata for the EIC with realistic uncertainties. The analysis follows the lines of those in Refs. [8,9], however, with updated kinematics and improved uncertainty estimates. Different from [8], which relied on DSSV08 helicity distributions as theory input
$[10,11]$, we start from the DSSV14 results to generate the observables that are subsequently smeared according to the expected experimental errors. We also incorporate semiinclusive estimates that were not discussed in [9] and study their impact on the quark helicity distributions. The analysis is carried out within the Monte Carlo sampling framework to estimate uncertainties, as introduced in [12]. The method relies on the generation of a set of replicas that are assumed to give a faithful representation of the underlying probability distribution of the helicity parton densities and helps as an alternative to the Lagrange multiplier approach used in $[8,9]$, which avoids the explicit adoption of tolerances, and also allows reweighting for different datasets.

Rather than to reweight the DSSV14 replicas obtained in [12], we generate a new set already incorporating the pseudodata corresponding to the inclusive DIS measurements at the lowest center of mass system (c.m.s.) energy configuration $(\sqrt{s}=45 \mathrm{GeV})$, which presumably will be one of the first results obtained at the EIC. The main motivation for this is that the reweighting of the replicas obtained in [12] with EIC pseudodata yields a very small effective number of replicas, which compromises the statistical rigor of the results. This feature is expected when the precision of the new data is significantly better than the one in the original fit or it probes rather different kinematics, as is the case for EIC data. Instead, with a new set of replicas that already includes some of the projected EIC measurements, we enhance the number of surviving replicas after subsequent reweighting with other pseudodata and make the sample large enough to derive statistically meaningful results and to discuss and compare their specific impact.

As result of the above mentioned procedure, we find that inclusive electron-proton data at the lowest energy configuration not only constrain significantly the gluon polarization down to $x \sim 10^{-4}$, as expected, but have also a sizable impact up to large values of $x$, thus constraining also the net polarization of the gluons in the region of momentum fractions were it is expected to get its most significant contributions. The total quark contribution $\Delta \Sigma$ is also significantly constrained compared to the original DSSV14 results. However, there is only a marginal impact on the polarization of the sea quarks. Adding the inclusive electron-proton DIS data at the higher c.m.s. energy $(\sqrt{s}=141 \mathrm{GeV})$ roughly duplicates the impact on the gluon polarization uncertainty and extends the constraints one decade further in the parton momentum fraction. In this way the EIC would probe partons carrying down to a hundred thousandth of the proton momentum. Semiinclusive data complement inclusive measurements and achieve flavor separation between quarks and antiquarks. In a similar way, electron-helium inclusive measurements complement inclusive proton ones, pushing the constraints on the total quark polarizations for the different flavors to an unprecedented level. 
The remainder of the paper is organized as follows: in the next section we discuss the generation of the different pseudodata for the inclusive and semi-inclusive measurements used in our analysis, and their corresponding uncertainty estimates. We also present in that section an assessment of the kinematical dependence of the impact one could anticipate studying the correlation and sensitivity coefficients for the most relevant quantities. Next, we briefly discuss the way in which we produce helicity distribution replicas from the DSSV14 dataset supplemented with the low c.m.s. energy electron-proton inclusive DIS pseudodata. In Sec. IV, we discuss the impact of both lower and higher c.m.s. energy inclusive pseudodatasets and compare with the original DSSV14 estimates. We also present the impact of the semi-inclusive data, and of the electron-helium measurements, respectively. Finally, we summarize our results and present our conclusions.

\section{PSEUDODATA FOR POLARIZED DIS AND SIDIS AT THE EIC}

\section{A. Generation of pseudodata}

To quantify the impact of an EIC on our understanding of helicity parton distribution functions (PDFs), we generate sets of pseudodata for lepton proton and lepton Helium-3 scattering at center-of-mass energies of $\sqrt{s}=44.7 \mathrm{GeV}$, $141.4 \mathrm{GeV}$, and $115.2 \mathrm{GeV}$, respectively.

We use the Polarised Electron Proton Scattering Interactions (PEPSI) Monte Carlo (MC) generator [13] to produce pseudodata at the EIC kinematics for the inclusive and semi-inclusive DIS of longitudinally polarized electrons and protons with identified charged pions and kaons in the final state and estimate in this way the statistical accuracy of the datasets. In a second step we randomize the full NLO theoretical estimates based on DSSV helicity densities [4] and DSS fragmentation functions $[14,15]$, according to the estimated statistical accuracy. The PEPSI generator is, at present, the only generator available for integrating longitudinal spin effects consistently for quarks and gluons and capable of producing longitudinally polarized SIDIS events.

For the generated events, we demand a minimum $Q^{2}$ of $1 \mathrm{GeV}^{2}$, a squared invariant mass of the virtual photonproton system $W^{2}$ larger than $10 \mathrm{GeV}^{2}$, and $0.01 \leq$ $y \leq 0.95$, where $y, Q^{2}, W^{2}$ and $x$ are the usual DIS kinematical variables, defined in terms of the nucleon, the photon, and the incoming electron four-momenta $p, q$ and $k$, respectively, as follows:

$y=\frac{q \cdot p}{k \cdot p}, \quad Q^{2}=-q^{2}, \quad W^{2}=(p+q)^{2}, \quad x=\frac{Q^{2}}{2 p \cdot q}$.

The range of $y$ is further restricted from below by constraining the depolarization factor of the virtual photon

$$
D(y)=\frac{y(y-2)}{y^{2}+2(1-y)(1+R)}
$$

to be larger than 0.1 . Note that $R$ denotes the ratio of the longitudinal to transverse virtual photon cross sections. To ensure detection of the scattered lepton, we require a minimum momentum of $0.5 \mathrm{GeV}$ and, in the case of SIDIS, only hadrons with a momentum $p_{H}$ larger than $1 \mathrm{GeV}$ and a fractional energy in the range $0.2 \leq z \leq 0.8$ are accepted, with $z$ defined as

$$
z=\frac{p_{H} \cdot p}{p \cdot q}
$$

Final state particles are detected within four units in rapidity in both directions $-4<\eta<4$, corresponding to a separation of at least 2 degrees from the beam line. The statistical accuracy of each DIS and SIDIS dataset corresponds to a accumulated integrated luminosity of $10 \mathrm{fb}^{-1}$.

The PEPSI MC allows one to generate events with definite helicities of the colliding lepton and proton beams, i.e., to study the longitudinal double-spin asymmetry. Note that

$$
\begin{gathered}
A_{\|}\left(x, Q^{2}\right)=\frac{d \sigma^{++}-d \sigma^{+-}}{d \sigma^{++}+d \sigma^{+-}} \\
=D(y) \frac{g_{1}\left(x, Q^{2}\right)}{F_{1}\left(x, Q^{2}\right)},
\end{gathered}
$$

which is related to the ratio of virtual photoabsorption cross sections, expressed by DIS structure functions in (5), through the depolarization factor $D(y)$. In (5), and also in (2), we have neglected kinematic corrections proportional to $\gamma=\sqrt{4 M^{2} x^{2} / Q^{2}}$, with $M$ the proton mass, which are negligible at a collider. Potentially problematic low values of $Q^{2}$ imply also rather low values of $x$, as shown in Figs. 1-3.

While containing spin-dependent hard scattering matrix elements at $\mathcal{O}\left(\alpha_{\mathrm{s}}\right)$ accuracy, the PEPSI MC is not capable of simulating parton showers, which properly track the polarization of the partons involved, and hence this option has been turned off for generating the EIC data. QED radiative corrections are known to be sizable and complicate the determination of the "true" values of $x$ and $Q^{2}$. We do not consider QED radiative corrections to be a major limitation on proposed DIS and SIDIS measurements at an EIC as unfolding their effects on the measurements, as has become standard at lepton-hadron experiments, i.e., H1, Zeus, HERMES, COMPASS. Available MC tools [16] will be further refined in the upcoming years; there is already a significant ongoing effort to implement QED radiative effects also in the general purpose generators, i.e., PYTHIA-8 [17]. 


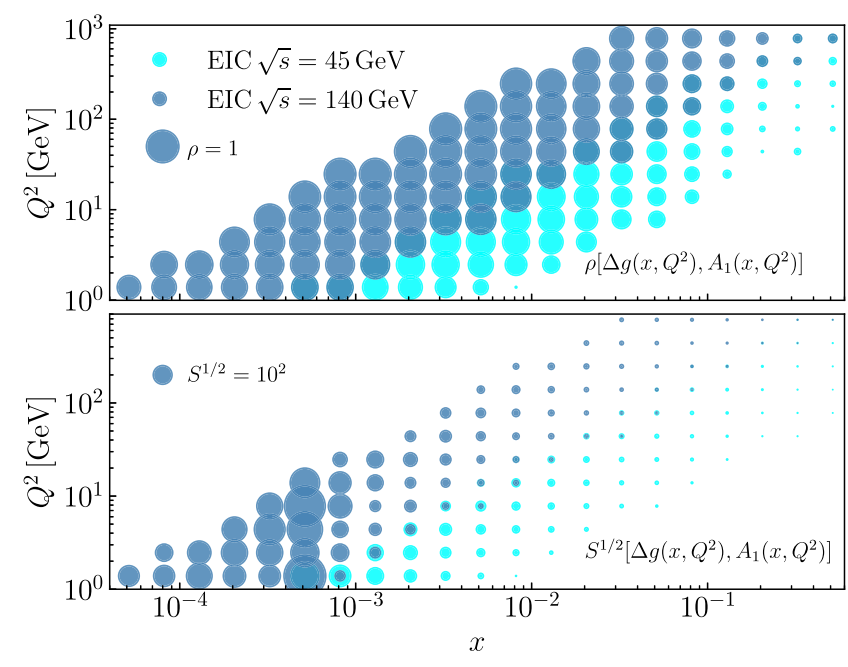

FIG. 1. Correlation (upper panel) and sensitivity (lower panel) coefficients between the gluon helicity distribution $\Delta g\left(x, Q^{2}\right)$ and the double spin asymmetry $A_{1}$, as a function of $\left\{x, Q^{2}\right\}$. The light blue and blue circles represent the value of the correlation (sensitivity) coefficient for $\sqrt{s}=45 \mathrm{GeV}$ and $\sqrt{s}=140 \mathrm{GeV}$, respectively. In all the cases the size of the circles is proportional to the value of the correlation (sensitivity) coefficient.

As in Ref. [9], the actual pseudodata used in our analyses below are not the generated ones but theoretical estimates of the spin asymmetries at NLO accuracy based on the latest DSSV helicity densities [4] and fragmentation functions $[14,15]$, reflecting the same relative statistical accuracy in each $x, Q^{2}$ bin as the Monte Carlo data, and having their central values randomized within one-sigma uncertainties. In our simulations of DIS and SIDIS off polarized neutrons, we assume that the experiment uses a polarized ${ }^{3} \mathrm{He}$ beam. To ensure that the scattering happened on the neutron, one requires the spectator protons to be tagged, a commonly used technique. For the EIC, great care is taken to integrate detectors along the outgoing hadron beam into the wider interaction region detecting spectator protons, protons from diffractive reactions and nuclear breakup and to guarantee high detection efficiencies of $>80 \%$. The size of the asymmetry sets the scale at which one needs to control systematic uncertainties due to detector performance or luminosity measurements. Until the EIC detector design is finalized, the approach taken for the systematic uncertainties is to assume that one can reach what was achieved at HERA, for inclusive measurements $1.6 \%$ and for the SIDIS measurements $3.5 \%$ point-to-point systematic uncertainty is assumed. In addition, a scale uncertainty due to the luminosity and lepton and hadron polarization measurement of $2.3 \%$ has been assigned. We have checked that adding up to a $2 \%$ systematic uncertainty to DIS pseudodata and $3.5 \%$ to SIDIS, respectively, do not have a significant impact in our estimates. This mainly happens because the most significant contributions to the effective $\chi^{2}$ minimized to obtain the replicas and to compute the new weights come, both for DIS and SIDIS, from low- $x$ pseudodata points, where the effects of systematic uncertainties is diluted. In addition, for SIDIS at higher values of $x$, the uncertainties are dominated by those of the fragmentation functions.

\section{B. Kinematical survey}

Our limited knowledge of the proton spin budget comes mostly from the rather limited kinematical coverage of the present spin dependent world data, the anticipated wide kinematical range projected for the EIC is of critical importance. It is very enlightening to start assessing the impact of the future EIC data studying the correlation and sensitivity coefficients $[7,18,19]$ between the most representative helicity parton densities and the measured spin asymmetries, as a function of $x$ and $Q^{2}$.

While the correlation coefficients indicate the regions of the phase space where an observable is expected to provide the strongest constraints on the partonic densities [18], the ultimate impact on those distributions will also be determined by the present understanding of that observable. Therefore, it is instructive to analyze also the sensitivity coefficients [19] defined as a rescaled correlation that also accounts for the relation between the precision of the measurement and the current level of uncertainty of the parton density, which in this particular case is assumed to be given by the DSSV14 set. A more detailed discussion of the usefulness of these tools, as well as their applications to semi-inclusive data, can be found in [7] and references therein.

A major advantage of Monte Carlo sampling and using parton density replicas is the straightforward calculation of correlation and sensitivity coefficients between any projected measurement and a given parton distribution. The correlation coefficient $\rho\left[f_{i}, \mathcal{O}\right]$ between a parton density for a given flavor $i$ and an observable $\mathcal{O}$ (i.e., a polarized asymmetry) is defined as [18] follows:

$$
\rho\left[f_{i}, \mathcal{O}\right]=\frac{\left\langle\mathcal{O} \cdot f_{i}\right\rangle-\langle\mathcal{O}\rangle\left\langle f_{i}\right\rangle}{\Delta \mathcal{O} \Delta f_{i}},
$$

where the mean values are calculated over the ensemble of replicas as

$$
\langle\mathcal{O}\rangle=\frac{1}{N} \sum_{k=1}^{N} \mathcal{O}\left[f_{i}^{(k)}\right],
$$

with $N$ being the number of replicas and the standard deviation for the observable and parton density given by

$$
\Delta \mathcal{O}=\sqrt{\frac{1}{N-1} \sum_{k=1}^{N}\left(\mathcal{O}\left[f_{i}^{(k)}\right]-\langle\mathcal{O}\rangle\right)^{2}} .
$$


Values for $|\rho|$ close to unity indicate that the observable and the parton density are highly correlated and, therefore, the inclusion of these particular data with a competitive experimental uncertainty could be able to further constrain the parton density. Values close to zero are obtained for uncorrelated observables and would never be able to improve the parton density determination, irrespective of the precision of the data. For simplicity, we omitted the dependencies on $x, Q^{2}$, and $z$, however, the correlation coefficients are defined for the kinematics of each particular (pseudo)data point, allowing a straightforward comparison between the constraining power of different kinematics regions. The sensitivity coefficient [19] on the other hand are given by

$$
S\left[f_{i}, \mathcal{O}\right]=\frac{\left\langle\mathcal{O} \cdot f_{i}\right\rangle-\langle\mathcal{O}\rangle\left\langle f_{i}\right\rangle}{\xi \Delta \mathcal{O} \Delta f_{i}},
$$

where the scaling factor

$$
\xi \equiv \frac{\delta \mathcal{O}}{\Delta \mathcal{O}}
$$

is defined as the ratio of the experimental measurement uncertainty $\delta \mathcal{O}$, and the theoretical uncertainty for the same observable propagated from the parton density $\Delta \mathcal{O}$. The scaled correlation coefficient suppresses those regions of the phase space for which the experimental uncertainty is large compared to the uncertainty associated to the PDFs while it enhances those regions where the relation is inverted, meaning where the biggest impact on the distributions is expected.

Since inclusive photon mediated DIS data with proton beams by itself have no charge separation power, but a limited flavor separation via the scale dependence, one expects to mainly determine the flavor singlet combination $\Delta \Sigma=\sum_{q=u, d, s}(\Delta q+\Delta \bar{q})$ and the gluon-helicity distribution $\Delta g$. Consequently, we focus first on those helicity densities.

In Figs. 1 and 2 we show the correlation (upper panels) and squared sensitivity (lower panels) coefficients between the gluon helicity distribution $\Delta g$ and the inclusive spin asymmetries $A_{1}$, as well as the correlation (squared sensitivity) between the flavor singlet helicity distribution $\Delta \Sigma$ and $A_{1}$, as a function of $x$ and $Q^{2}$, for both of the c.m.s. energies under consideration, $\sqrt{s}=44.7$ and $141.4 \mathrm{GeV}$. The inclusive and semi-inclusive asymmetries $A_{1}$ and $A_{1}^{h}$ can be written as in Eq. (4), with a depolarization factor equal to 1 , that is,

$A_{1}\left(x, Q^{2}\right)=\frac{g_{1}\left(x, Q^{2}\right)}{F_{1}\left(x, Q^{2}\right)}, \quad A_{1}^{h}\left(x, Q^{2}\right)=\frac{g_{1}^{h}\left(x, Q^{2}\right)}{F_{1}^{h}\left(x, Q^{2}\right)}$,

where $g_{1}, F_{1}$ and $g_{1}^{h}, F_{1}^{h}$ are the inclusive and semiinclusive longitudinally polarized and unpolarized structure

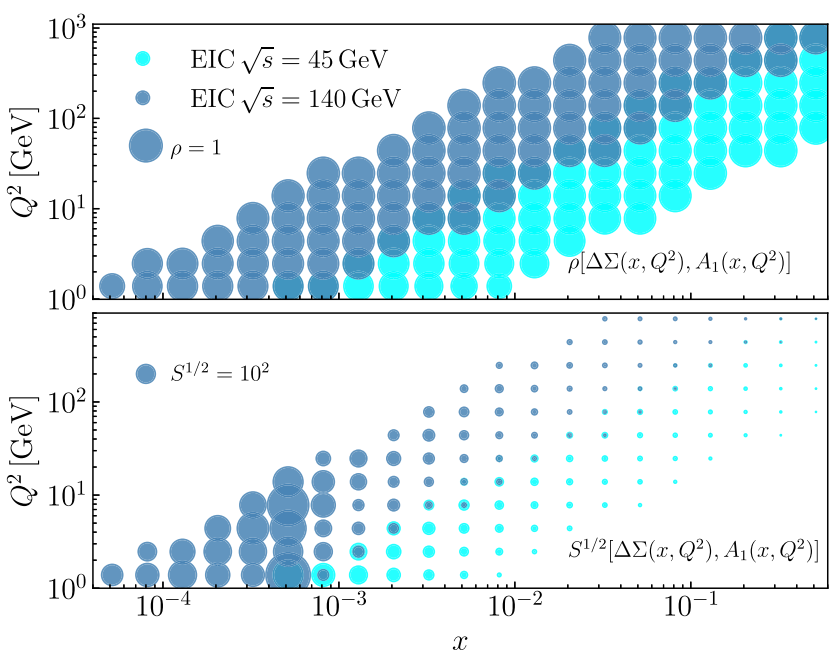

FIG. 2. Same as Fig. 1, for the quark flavor singlet helicity distribution, $\Delta \Sigma\left(x, Q^{2}\right)$.

functions, respectively. In all the cases, the size of the circles is proportional to the value of the correlation (squared sensitivity) coefficient. A reference value is included in the plots for both coefficients. The upper panel of Fig. 1 shows that for both c.m.s. energy configurations there are strong correlations between the inclusive asymmetries and the gluon helicity, however, the higher energy configuration covers one decade further in momentum fraction $x$. On the other hand, in the upper right corner of the plot, which corresponds to larger values of $x$ an $Q^{2}$, the correlation fades away faster for the lower energy configuration, reducing the grid of values over which the scale violations are effectively probed and suggesting a weaker impact. Focusing on the lower panel of Fig. 1, the sensitivity plot shows that the lower left corner, mostly missed by the low energy configuration, is precisely where there is more potential for improving the gluon distribution. Notice that higher values of $x$ are already covered by the RHIC measurements, and to a lesser extent by fixed target DIS experiments.

For the singlet helicity distribution $\Delta \Sigma$, the correlation and sensitivity coefficients show similar features, however, at variance with what happens for the gluons, the correlation with the singlet remains strong in the upper right corner of the plot, due to the dominant quark contribution to the asymmetry at higher values of momentum fractions. In this way we do not anticipate a significant difference in the constraint on $\Delta \Sigma$ coming from either energy configurations, except at very low values of $x$, only probed by the large c.m.s. energy configuration.

As we explain in more detail in Sec. IV, inclusive DIS asymmetries cannot discriminate between quark and antiquarks, and therefore, are unable to constrain by themselves the sea quark polarization, unless a very strong spin-flavor symmetry assumption is invoked. SIDIS asymmetries, on the other hand, weight the individual sea quarks 

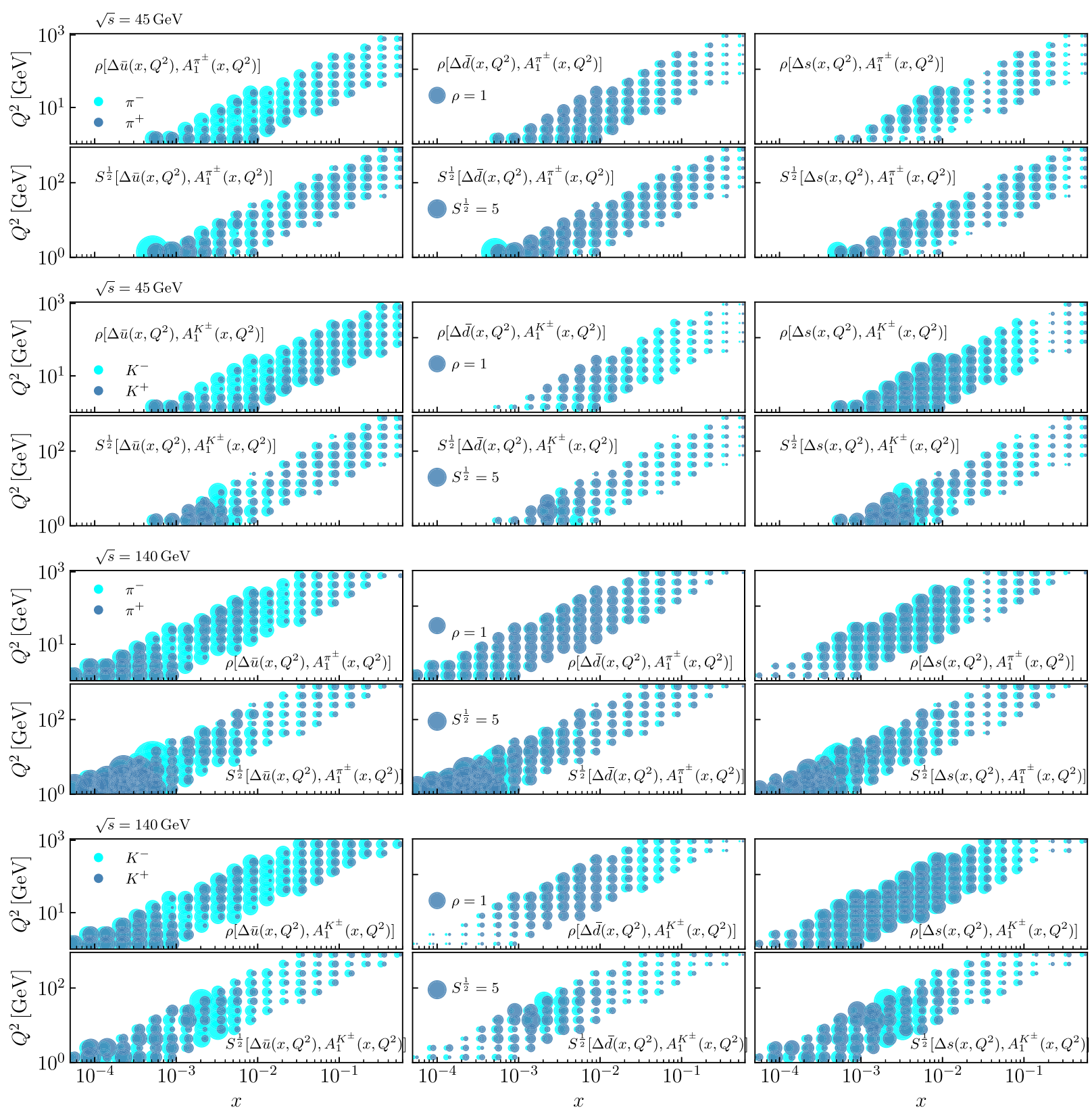

FIG. 3. Correlation and sensitivity coefficients between the sea quark helicity distributions and the pion and kaon SIDIS asymmetries as a function of $\left\{x, Q^{2}\right\}$. The light blue and blue circles represent the value of the correlation (sensitivity) coefficient for negatively and positively charged hadrons, respectively. In all the cases the size of the circles is proportional to the value of the correlation (sensitivity) coefficient. The four upper rows correspond to $\sqrt{s}=44.7 \mathrm{GeV}$ SIDIS pseudodata, and the lower rows to $\sqrt{s}=141.4 \mathrm{GeV}$, respectively.

contributions differently through their respective hadronization probabilities into specific final-state charged hadrons. In Fig. 3 we show, precisely, the estimates for the correlation and sensitivity coefficients between the sea quark helicity distribution and the pion and kaon SIDIS spin asymmetries reflecting these features.

Regarding the correlation plots for pion asymmetries, in the panels of the upper row in Fig. 3, it is quite apparent that while $A_{1}^{\pi^{-}}$is sensitive to $\bar{u}$ quarks, $A_{1}^{\pi^{+}}$is sensitive to $\bar{d}$ quarks, being consistent with the valence quarks of the pion. The correlations are stronger at lower values of momentum fraction, where sea quark contributions dominate over those of the valence quark in the proton. Both pion asymmetries show a weaker but still significant correlation with strange quarks. The final impact of the measurements depends, of course, on a subtle balance between the uncertainties of the pseudodata, the theory uncertainties inherent to the analysis, dominated in this 
case by the fragmentation functions, and our present knowledge on the sea quark polarization. This last feature is quantified by the sensitivity coefficients, shown in the second row panels of Fig. 3, and indeed suggest that a very significant impact should be expected. Notice that these estimates are based on our present knowledge of fragmentation functions. Since unpolarized SIDIS measurements at the EIC have been shown to improve significantly the determination of fragmentation functions [7], the present estimates should be taken as rather conservative.

In the case of charged kaon SIDIS asymmetries, the panels of the third row of Fig. 3 show that the strongest correlations are found with strange quarks, to a lesser extent with $\bar{u}$ quarks and least with $\bar{d}$ quarks. This hierarchy can be again traced back to the parton composition of charged kaons and to the implicit assumption in this analysis of the same polarization for strange quarks and antiquarks. Finally, in terms of the sensitivity, the fourth row of panels of Fig. 3 displays that while the kaon SIDIS asymmetries have typically weaker impact on the $\bar{u}$ and $\bar{d}$ quarks than those with pions in final state, they have a much stronger sensitivity on the strange quark polarization.

The remaining rows in Fig. 3 show the same as the first 4 rows but for $\sqrt{s}=141.4 \mathrm{GeV}$ SIDIS data. The main difference with the lower c.m.s. energy configuration is, as in the inclusive case, that the data explore one decade lower in the parton momentum fraction, where the largest impact is expected. This feature, however, happens only for valence quarks in the respective final-state hadron, as shown both by the correlation and the sensitivity estimates, but the effect is weaker for unfavored quark types.

\section{MONTE CARLO SAMPLING OF HELICITY PARTON DISTRIBUTIONS}

In order to investigate the impact of the EIC data, as usual we implement a NLO global analysis combining the datasets coming from different spin dependent experiments performed so far with the pseudodata discussed in the previous section. However, rather than using the Lagrange multiplier methodology to estimate uncertainties as in our previous assessments $[8,9]$, in the following we implement a Monte Carlo sampling strategy with predetermined functional forms for the helicity densities, along the lines of reference [12], to which we refer for more specific details and discussions. The major advantage of this approach is to combine the computational power of the Mellin transform technique with the statistical tools provided by Monte Carlo sampling. Schematically, the strategy is to generate in a first step a Monte Carlo ensemble of replicas of the original data (and pseudodata in the present case) with a probability distribution derived from the reported/expected uncertainties of each experiment within the desired accuracy [20,21]. In a subsequent step, a set of parton densities is obtained for each replica of the data with the standard fitting techniques. The ensemble of corresponding parton densities replicas obtained in this way is expected to encode all the information relevant to determine the parton distribution. For instance, the central value of the parton densities, or any quantity derived from them, is taken to be the average over the parton density replicas, and the corresponding uncertainty is the statistical standard deviation. In this way, the Monte Carlo sampling strategy avoids introducing a tolerance criterion to estimate uncertainties, and other known shortcomings in the propagation of uncertainties to experimental observables, characteristic to the Lagrange multiplier method [22] and the Hessian approach [23], respectively. In the case of the Lagrange multiplier method, in addition to the choice of the tolerance, the main difficulty consists in that the computation of the uncertainty band for a given observable even at a single kinematical point requires performing a number of new fits, including the evaluation of the observable for different Lagrange multiplier values in the minimization function of the fit until the chosen tolerance is reached [22]. In the case of the Hessian approach, the main obstacle arises when the $\chi^{2}$ profile of the fit shows a nonparabolic behavior, as assumed by the method. This is precisely what has been found in helicity distributions fits in Ref. [11]. As we mentioned previously, within the Monte Carlo approach, there is no need to choose a tolerance, and the propagation of errors is accomplished evaluating the desired observable over the set of replicas and computing its standard deviation.

The Monte Carlo sampling strategy provides also a very powerful tool to assess and compare the impact of different datasets in the determination of parton densities, known as reweighting. The reweighting technique [24,25] allows to incorporate the information provided by a new set of data into an existing ensemble of parton density replicas without the need of refitting but preserving the statistical rigor of its extraction. The method has already been successfully demonstrated in the context of the extraction of parton densities, see, for instance, Refs. [5,12,26-28]. By means of Bayesian inference, it is possible to modify the original probability distribution of an ensemble of parton density replicas to account for the information contained in a new measurement [24]. This is implemented assigning a new weight to each replica, which measures its consistency with the new data.

The Bayesian reweighting is equivalent to a refit including the additional set of data, as long as the impact of the new data is not too significant, for instance, by constraining some aspect of the parton densities that was largely undetermined before. Such a scenario would lead to a very large number of replicas with essentially vanishing weights making a full refit inevitable. This is precisely the situation of the EIC pseudodata since they extend by one or two additional decades in parton momentum fraction, respectively, as shown in the previous section. For this reason, rather than reweighting the DSSV14 replicas obtained in [12], we first produce a new set of replicas sampling the 


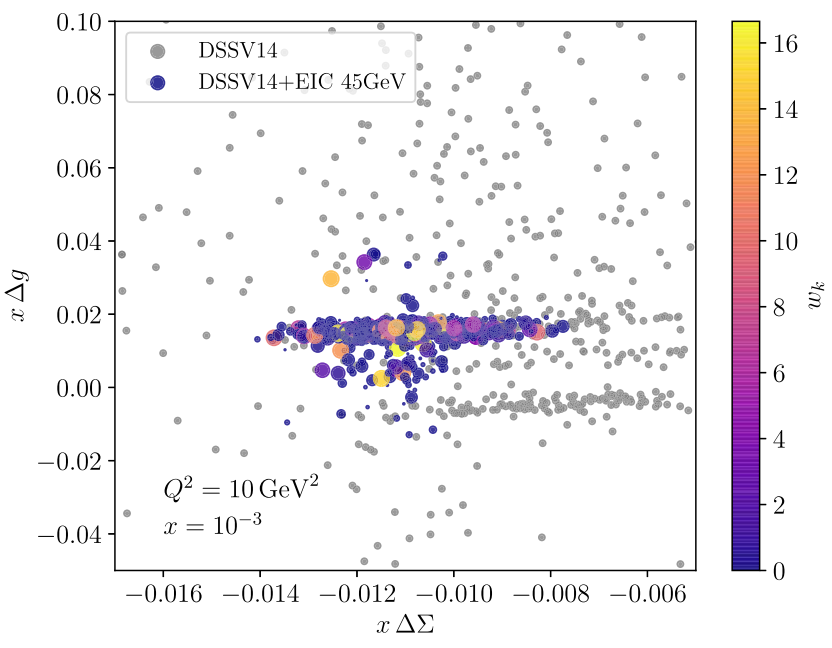

FIG. 4. Values for $x \Delta g$ and $x \Delta \Sigma$ at $x=0.001$ and $Q^{2}=$ $10 \mathrm{GeV}^{2}$ for the DSSV14 replicas (grey dots) and those of the new set that includes EIC inclusive DIS electron-proton pseudodata at $\sqrt{s}=44.7 \mathrm{GeV}$ (in color). The size and color of the new replicas represents the weight they obtain by reweighting with EIC $\sqrt{s}=141.4 \mathrm{GeV}$ pseudodata.

original DSSV14 dataset supplemented with the inclusive DIS electron-proton pseudodata at $\sqrt{s}=44.7 \mathrm{GeV}$.

In Fig. 4 we present by the grey dots the results of the gluon helicity $\Delta g$ and the quark flavor singlet $\Delta \Sigma$ at $x=$ 0.001 at $Q^{2}=10 \mathrm{GeV}^{2}$ as obtained from the DSSV14 replicas. The replicas are rather scattered over a comparatively large range of values of $\Delta g$ and $\Delta \Sigma$ that are representative of the corresponding uncertainties in both distributions. The colored dots represent the replicas obtained adding inclusive electron-proton DIS pseudodata at $\sqrt{s}=44.7 \mathrm{GeV}$. The new replicas are clustered in a much more restricted area, which is the range of values that is the relevant one once the EIC data are included. Most of the original replicas become irrelevant, and those in the relevant region are extremely few in number. Additionally, the size and color of the new replicas represents the weight they obtain by reweighting with EIC inclusive DIS electron-proton pseudodata at $\sqrt{s}=141.4 \mathrm{GeV}$.

In Fig. 5 we show the same replicas as in Fig. 4 but now in terms of the truncated moments $\Delta g$ and $\Delta \Sigma$, which represent the net contribution to the proton spin arising from the spin of quarks and gluons with momentum fractions larger than $x=10^{-5}$. These moments collect contributions not only from small $x$ but mostly from larger momentum fractions, if one assumes the low- $x$ extrapolation of the DSSV14 helicity distributions, as shown in Ref. [9]. The ellipses denote the $1-\sigma$ limits for the values associated to the new replicas and their reweighting with DIS pseudodata at $\sqrt{s}=141.4 \mathrm{GeV}$. Notice that the reweighting procedure reduces the number of effective replicas to $N_{\text {eff }}=82$ from the 950 of the initial set.

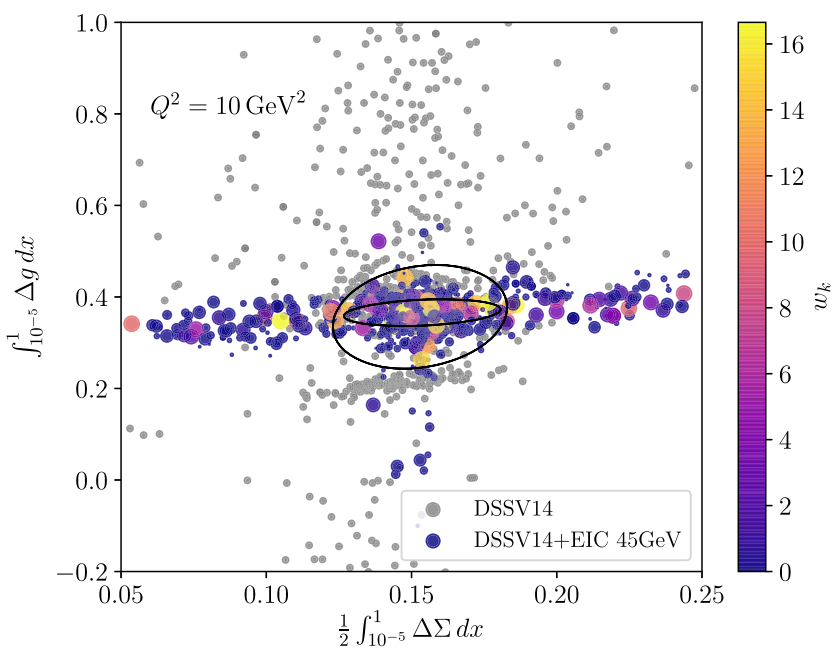

FIG. 5. Values for the moments $\Delta g$ and $\Delta \Sigma$ integrated between $x=10^{-5}$ and 1 at $Q^{2}=10 \mathrm{GeV}^{2}$ for the DSSV14 replicas (grey dots) and those of the new set that includes EIC inclusive DIS electron-proton pseudodata at $\sqrt{s}=44.7 \mathrm{GeV}$ (in color). The size and color of the new replicas represents the weight obtained by reweighting with the EIC $\sqrt{s}=141.4 \mathrm{GeV}$ pseudodata. The ellipses denote the $1-\sigma$ limits for the values associated to the new replicas and their reweighting with DIS pseudodata at $\sqrt{s}=141.4 \mathrm{GeV}$.

Since we expect a much better constraining power coming from the EIC pseudodata at $x<0.001$, as in Ref. [12], we have further increased the flexibility of the parametrizations relative to that of the original DSSV14 set [4] and enhanced the parameter sampling strategy to guarantee that no significant bias is introduced in the region constrained by the data, and that uniform probability distributions are obtained in the unmeasured region.

The DSSV analyses $[4,10,11]$ adopt the most traditional fitting approach at NLO accuracy assuming a flexible functional form to parametrize the helicity PDFs as functions of the parton momentum fraction $x$ at an initial scale of $\mu_{0}=1 \mathrm{GeV}$,

$$
x \Delta f_{i}\left(x, \mu_{0}\right)=N_{i} x^{\alpha_{i}}(1-x)^{\beta_{i}}\left(1+\gamma_{i} \sqrt{x}+\eta_{i} x^{\kappa_{i}}\right),
$$

where the label $i$ denotes different flavor combinations $\Delta u+\Delta \bar{u}, \Delta d+\Delta \bar{d}, \Delta \bar{u}, \Delta \bar{d}, \Delta \bar{s} \equiv \Delta s$ and the gluon density $\Delta g$. As usual, $\Delta f_{i}$ represents the difference of densities with parton spins aligned and antialigned with the spin of the parent proton. The optimization of the fit to data is carried out by varying the set of fit parameters $\left\{a_{i}\right\}=$ $\left\{N_{i}, \alpha_{i}, \beta_{i}, \gamma_{i}, \eta_{i}, \kappa_{i}\right\}$ iteratively, as long as a minimum in the effective $\chi^{2}$ function is reached. In each iteration the PDFs are evolved to the scale $\mu>\mu_{0}$ relevant in the experiment and used to compute the corresponding observables and the effective $\chi^{2}$ function to be minimized. Equivalently, Eq. (12) can be rewritten to 


$$
x \Delta f_{i}\left(x, \mu_{0}\right)=\sum_{j=1}^{3} N_{i j} x^{\alpha_{i j}}(1-x)^{\beta_{i j}},
$$

specially suited for working in the Mellin representation [29] since each term is the integrand of an Euler integral of the first kind, and the corresponding moments are standard beta functions. Of course, some of the parameters in Eq. (13) are no longer independent. This parametrizations have been found to be flexible enough to describe the DSSV14 dataset in the sense that using more complex functional forms lead to equally good fits to data and also to statistically equivalent replicas of the data. Actually, the currently available data do not even fully constrain the values for the fit parameters, and some restrictions on the parameter space have to be imposed, reducing them to typically five free parameters per flavor or even less in the case of antiquarks, such that a unique and stable minimum in $\chi^{2}$ can be found. In spite of this flexibility in the region supported by the data, the values for the parameters that optimize the fit to data determine the extrapolation into the unmeasured region, mostly $x<0.001$, and constrain artificially the range of variation of the distributions. To avoid this problem, additional terms in Eq. (13) are added. The new parameters are chosen so that they only modify the unmeasured low- $x$ domain but leave the region constrained by the data unaffected, but it is required that the integrability of the parton densities and their convenient properties under Mellin transformations are preserved. Different from Ref. [12], which focused mainly on studying the already measured region $x>0.001$ and where only one of these additional terms was included for the gluon helicity distribution, for the new replicas we activate three additional low- $x$ terms per flavor $(j=6)$, allowing roughly a similar degree of flexibility in the so far unmeasured region $x<0.001$ as for the values of momentum fraction covered by the present data. More specifically, in the additional low- $x$ terms, the exponents $\beta_{i j}$ for $j=4,5,6$ driving the high- $x$ part of the parametrizations are fixed to an extremely large value $\left(\beta_{i j}=150\right)$ to guarantee that the contributions of these terms to the intermediate and large $x$ region are strongly suppressed. The $\beta_{i j}$ exponents already included in the standard parametrization $(j=1,2,3)$ are, in principle, free but in practice strongly constrained by the positivity criteria. In this way, the new parametrization can reproduce the shape of the DSSV14 set at $x>0.001$ but has a free low- $x$ behavior. On the other hand, the exponents $\alpha_{i j}$ driving the low $x$ behavior are, as usual, constrained so that the moments of the distributions converge, and the net polarization carried by each flavor remains finite. In all, the number of free parameters adds up to 46, against the 22 parameters of DSSV14.

In the following section we present the results of the new fit and the corresponding replica set, obtained combining the dataset of the DSSV14 analysis with the inclusive DIS electron-proton pseudodata at $\sqrt{s}=44.7 \mathrm{GeV}$. In order to assess the impact of the remaining EIC pseudodatasets, such as the SIDIS measurements at $\sqrt{s}=44.7 \mathrm{GeV}$ and inclusive DIS electron-proton and electron-helium at $\sqrt{s}=141.4$ and $\sqrt{s}=115.2 \mathrm{GeV}$, respectively, we then reweight the newly produced set of replicas. The outcome of these reweightings represents the combined impact of the first stage of the EIC together with the SIDIS measurements with a second energy stage and including the results of electron-helium collisions, respectively.

\section{RESULTS}

\section{A. Impact of deep inelastic scattering data}

Our results focusing on the impact of the inclusive DIS measurements to the gluon helicity through the corresponding constraints on the spin dependent structure function $g_{1}\left(x, Q^{2}\right)$ and its $Q^{2}$ dependence are described in the following. The totally inclusive photon mediated DIS cross section between longitudinally polarized leptons and nucleons can be written in terms of the structure function $g_{1}\left(x, Q^{2}\right)$, in full analogy to the structure function $F_{1}\left(x, Q^{2}\right)$, used in the case of the unpolarized cross section.

At NLO in QCD, the structure function $g_{1}\left(x, Q^{2}\right)$ is given in terms of the quark and gluon helicity densities $\Delta q\left(x, Q^{2}\right)$ and $\Delta g\left(x, Q^{2}\right)$ by

$$
\begin{aligned}
g_{1}\left(x, Q^{2}\right)= & \frac{1}{2} \sum_{q=u, d, s} e_{q}^{2}\{(\Delta q+\Delta \bar{q}) \\
& \left.+\frac{\alpha_{s}\left(Q^{2}\right)}{2 \pi}\left[\Delta C_{q} \otimes(\Delta q+\Delta \bar{q})+\Delta C_{g} \otimes \Delta g\right]\right\},
\end{aligned}
$$

where $\Delta C_{q}$ and $\Delta C_{g}$ are the spin dependent DIS coefficient functions [30], $\alpha_{s}\left(Q^{2}\right)$ is the QCD running coupling constant, and $\otimes$ stands for the usual convolution integral. Even though we perform our analysis at NLO, it is worth noticing that higher order QCD corrections to $g_{1}$ and its evolution have been computed at the two and three loop level, and including mass corrections [31-34]. Restricting ourselves to three quark flavors, the structure function can be alternatively written as follows:

$$
\begin{aligned}
g_{1}\left(x, Q^{2}\right)= & \left( \pm \frac{1}{12} \Delta q_{3}^{\mathrm{NS}}+\frac{1}{36} \Delta q_{8}^{\mathrm{NS}}+\frac{1}{9} \Delta \Sigma\right) \\
& \otimes\left(1+\frac{\alpha_{s}}{2 \pi} \Delta C_{q}\right)+\frac{\alpha_{s}}{2 \pi}\left(\sum_{q} e_{q}^{2}\right) \Delta C_{g} \otimes \Delta g
\end{aligned}
$$

where 


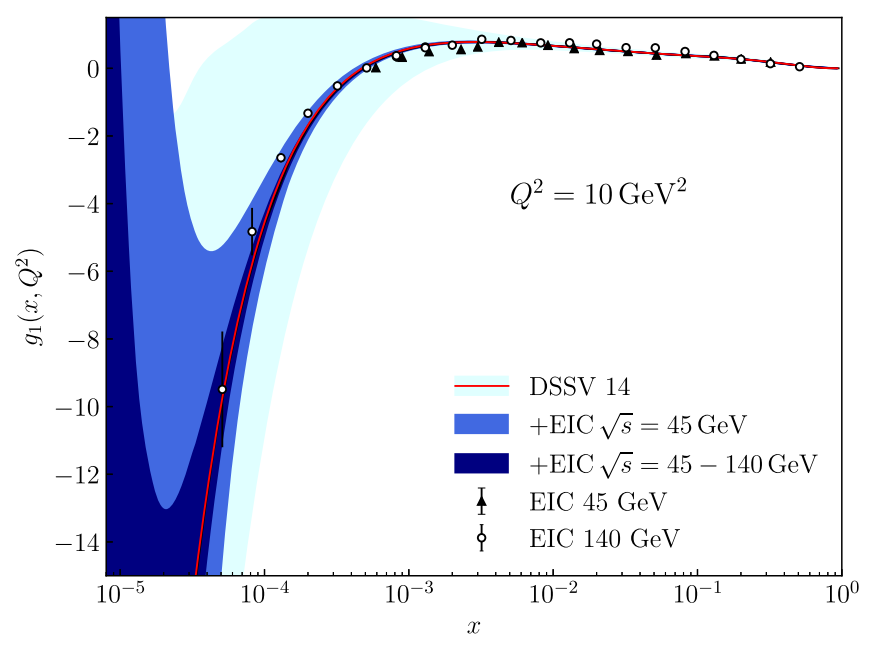

FIG. 6. Helicity structure function $g_{1}\left(x, Q^{2}\right)$ and its $68 \%$ C.L. band as a function of $x$ at $Q^{2}=10 \mathrm{GeV}^{2}$, calculated with the Monte Carlo variant of DSSV14. We include some the pseudodata points of $g_{1}$ for the two c.m.s. energies and their expected experimental uncertainties.

$$
\begin{aligned}
\Delta q_{3}^{\mathrm{NS}} & \equiv(\Delta u+\Delta \bar{u})-(\Delta d+\Delta \bar{d}) \\
\Delta q_{8}^{\mathrm{NS}} & \equiv(\Delta u+\Delta \bar{u})+(\Delta d+\Delta \bar{d})-2(\Delta s+\Delta \bar{s}) \\
\Delta \Sigma & \equiv(\Delta u+\Delta \bar{u})+(\Delta d+\Delta \bar{d})+(\Delta s+\Delta \bar{s}),
\end{aligned}
$$

and the \pm sign in Eq. (15) corresponds to scattering either a proton or a neutron, respectively. The first 2 nonsinglet distributions, $\Delta q_{3}^{\mathrm{NS}}$ and $\Delta q_{8}^{\mathrm{NS}}$, evolve independently in $Q^{2}$, whereas the gluon and the singlet distribution $\Delta \Sigma$ are coupled by the evolution equations.

It is worth noting that whereas $\Delta q_{3}^{\mathrm{NS}}$ could be obtained directly from data on a linear combination of the proton and the neutron (helium or deuterium) spin dependent structure functions, the remaining combinations in Eq. (15) and $\Delta g$ have to be obtained indirectly, through their different scale dependencies. The extended range both in $x$ and $Q^{2}$ of the data results that a much more precise determination of all the distributions can be achieved. However, it should be kept in mind that even with an unbounded set of inclusive data of unlimited precision, it would be impossible to disentangle $\Delta q$ from $\Delta \bar{q}$. The latter requires data from processes that weight quark and antiquarks differently, such as weak interactions or processes including hadronizations, like those probed by SIDIS, where the flavor content of the hadron observed in the final state can discriminate between quark and antiquark contributions.

In Fig. 6 we show the structure function $g_{1}\left(x, Q^{2}\right)$ as a function of $x$ and for a fixed value of $Q^{2}=10 \mathrm{GeV}^{2}$, computed at NLO accuracy using the Monte Carlo variant of the DSSV14 set of helicity distributions [12], which may be taken as representative of our present knowledge. The light cyan band represents its uncertainty for the $68 \%$ C.L. limit. The world data on $g_{1}$ is actually restricted to $x>$
0.004 and, consequently, below that threshold the DSSV14 expectation is just an extrapolation assuming that $\Delta q$ and $\Delta g$ vanish smoothly as $x \rightarrow 0$. The lack of data is reflected in the very rapid growth of the uncertainty band. Overlaid with the expectation for $g_{1}$, we also show some representative EIC pseudodata points, generated for both values of the c.m.s. energy analyzed in this study. The points are plotted just as a reference for the corresponding ranges in $x$ and for the size of the expected uncertainties. Notice that the pseudodata points occur at different values of $Q^{2}$; the selected points shown in the plot are those closest to $Q^{2}=10 \mathrm{GeV}^{2}$.

The light blue band in Fig. 6 shows the uncertainty in the structure function estimate when the inclusive DIS electron-proton pseudodata at $\sqrt{s}=44.7 \mathrm{GeV}$ are combined with the DSSV14 dataset and represent the comparative impact of the projected measurement. The darker blue band shows the effect of reweighting the replicas with DIS inclusive electron-proton pseudodata at $\sqrt{s}=141.4 \mathrm{GeV}$ and accounts for the combined effect of both pseudodatasets. The reweighting yields a number of effective replicas $N_{\text {eff }}=82$ to be compared with a total of 950 , which means a very significant impact. Finally, it is worth noticing that at very small values of $x$ the uncertainty estimate coming from the DSSV14 replicas happens to be smaller than those obtained with the new replicas and their reweighting. As it has been already pointed out above and in Ref. [12], the world data on $g_{1}$ that drive the behavior of the DSSV14 replicas is actually limited to $x>0.004$ and, consequently, below that threshold the DSSV14 expectation could be artificially restricted by the functional form assumed to extrapolate in the unmeasured region. On the other hand, the new replicas are based on a much more flexible parametrization, and their behavior is driven by pseudodata in a much more extended kinematical region.

Even though the exact scale dependence of $g_{1}$ is related to the size and evolution the whole set of helicity distributions in an extremely convoluted way, at small enough values of $x$, it is linked to the gluon helicity through the approximate relation:

$$
\frac{\partial g_{1}\left(x, Q^{2}\right)}{\partial \ln Q^{2}} \approx-\Delta g\left(x, Q^{2}\right),
$$

it simply states that for a given value of $x$ the larger is the gluon helicity, the steeper is the $Q^{2}$-dependence of $g_{1}\left(x, Q^{2}\right)$, and it gives a more intuitive picture of how an improved knowledge of $g_{1}$ at different scales constrains the gluon helicity.

In Fig. 7 we present the estimate for the logarithmic derivative of $g_{1}$ and the corresponding $68 \%$ C.L. limit uncertainty bands as a function of $Q^{2}$ for different values of $x$. The values for the logarithmic derivative of $g_{1}$ in Fig. 7 were estimated approximating the derivative as a ratio between the differences of two consecutive pseudodata 


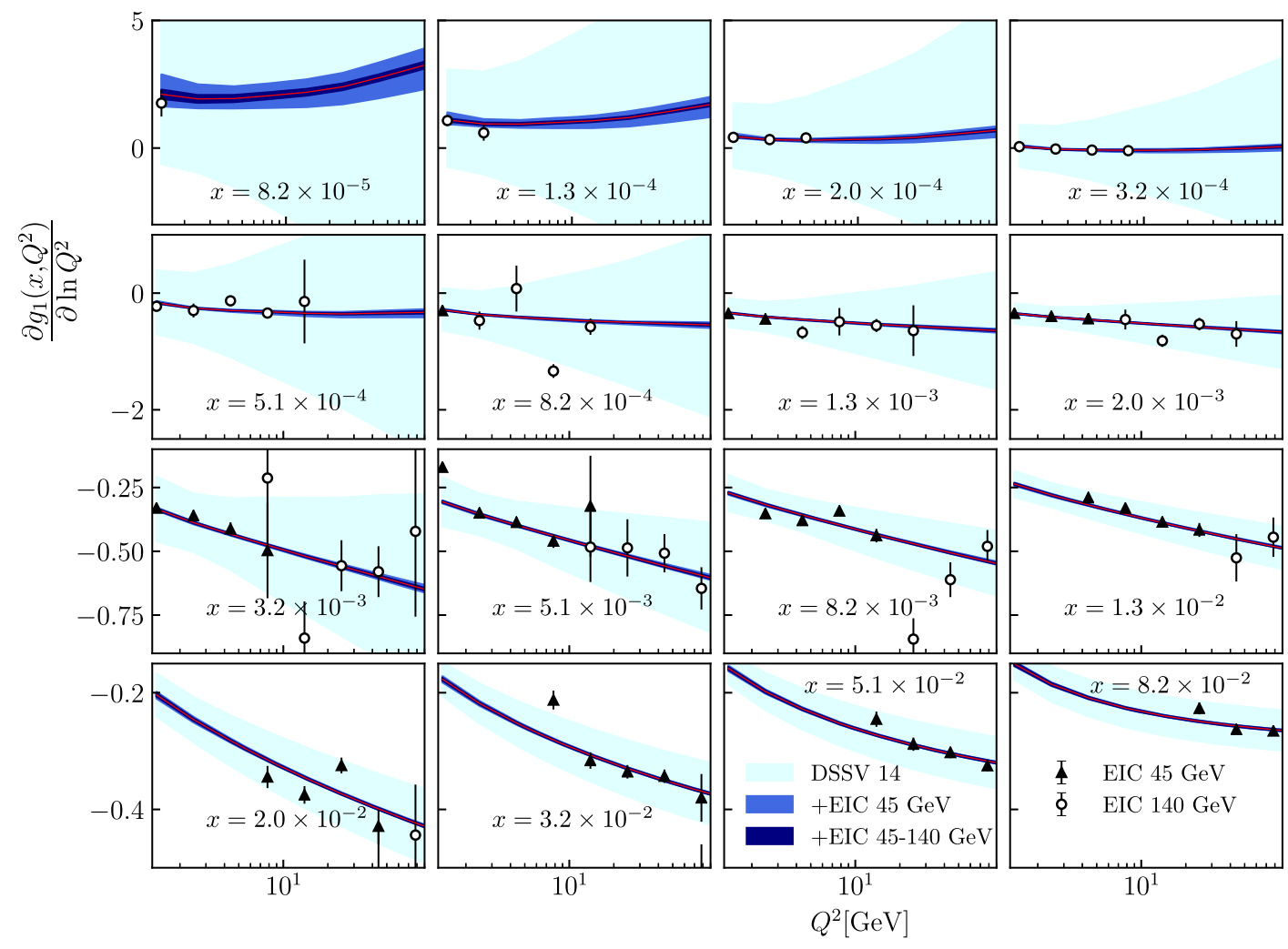

FIG. 7. Estimates for the logarithmic scaling violation of $g_{1}\left(x, Q^{2}\right)$ and the corresponding uncertainties, computed with the DSSV14 helicity parton densities, and the impact of including the DIS EIC pseudodatasets at $\sqrt{s}=44.7 \mathrm{GeV}$ and $\sqrt{s}=141.4 \mathrm{GeV}$, respectively.

points and the logarithms of their corresponding $Q^{2}$ values. Therefore, for each bin in $x$ it is necessary to have at least two pseudodata values for $g_{1}$ in different bins of $Q^{2}$. These approximations are used just to visualize the correlation. In the analysis we use always the full NLO evolution. Similarly to Fig. 6, we present the expectations derived from EIC pseudodata for the two values of $\sqrt{s}$, as well as the impact (in terms of the uncertainty bands) that those data points would have through the new replicas and their reweighting.

Let us make some remarks at this point. In the first place, and as expected, the uncertainty in the scaling violation grows dramatically in the DSSV14 estimate for lower values of $x$ due to the lack of data and, therefore, constraints to the gluon helicity for $x \lesssim 10^{-2}$. Second, the EIC pseudodata reduce considerably the range of variation allowed in the slope of $g_{1}$ and, consequently, the value for $\Delta g$. Finally, the difference in the $x$-range covered by the data for different c.m.s. energies is significantly different and, therefore, critical since the most important constraints on the gluon distribution are expected to come from the region, where the scaling violations are measured, as will be discussed below.

In terms of the helicity gluon distribution itself, the impact of the projected EIC data is even more graphic. In Fig. 8 we show the gluon helicity distribution and its uncertainty bands as a function of $x$ for $Q^{2}=10 \mathrm{GeV}^{2}$. The uncertainty estimates correspond to the standard deviation of the DSSV14 replicas (in light cyan), that of the replicas obtained combining the DSSV14 dataset and

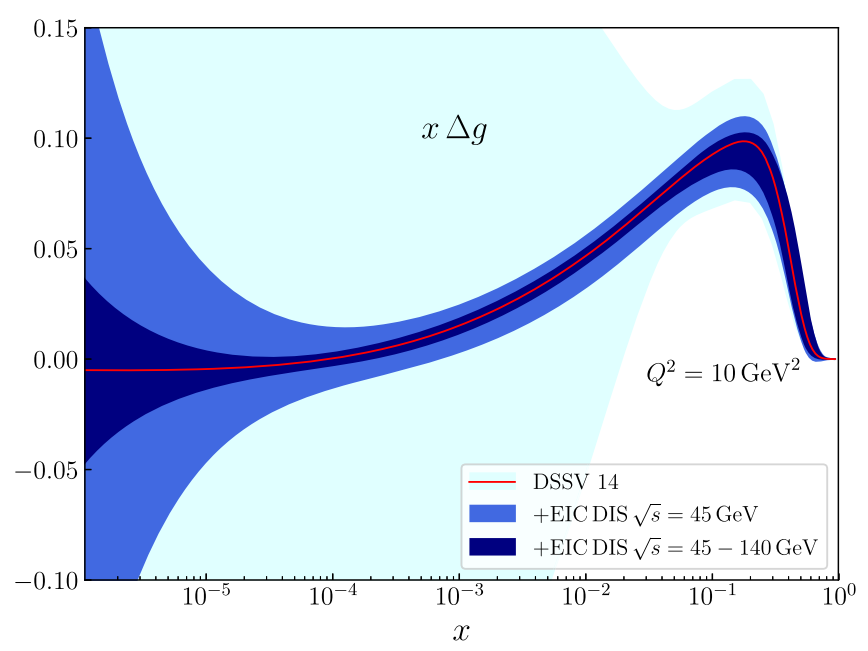

FIG. 8. Impact of the projected EIC DIS pseudodata on the gluon helicity distribution as a function of $x$ at $Q^{2}=10 \mathrm{GeV}^{2}$. Together with the DSSV14 estimate, we show the uncertainty bands resulting from the fit that includes the $\sqrt{s}=44.7$ DIS pseudodata and the reweighting with $\sqrt{s}=141.4 \mathrm{GeV}$ pseudodata. 


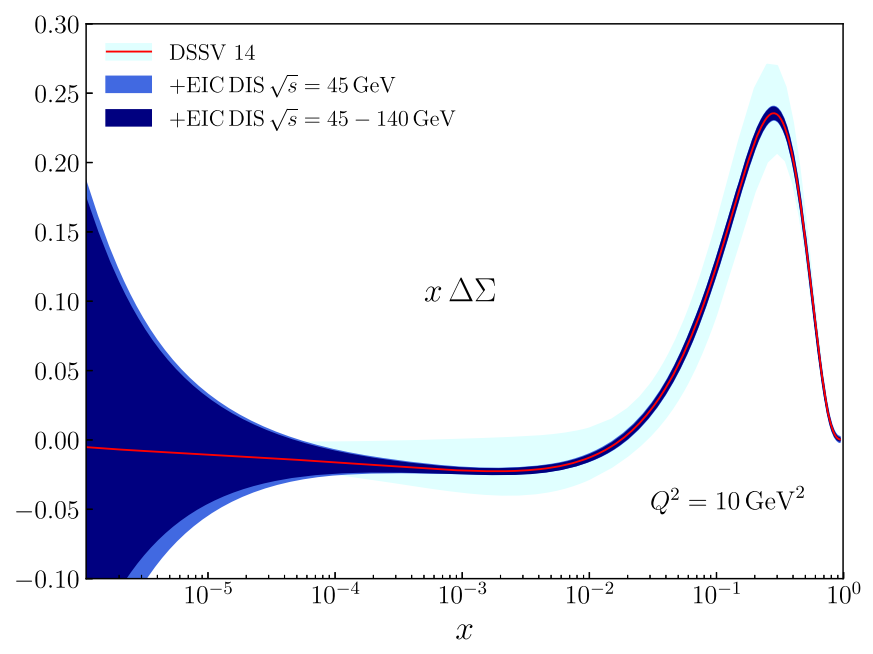

FIG. 9. The same as Fig. 8 but for the singlet quark helicity $\Delta \Sigma$ and its uncertainty bands.

the $\sqrt{s}=44.7 \mathrm{GeV}$ pseudodataset (light blue) and, after reweighting, the latter with the $\sqrt{s}=141.4 \mathrm{GeV}$ set (darker blue). As anticipated, the impact on the gluon helicity uncertainty is very significant, not only at very low values of momentum fraction where the DSSV14 is basically unconstrained, but also at intermediate values, where in spite of the availability of inclusive DIS data in the DSSV14 data, the gluons are still largely unconstrained. The impact of the combined high and low c.m.s. energy configurations is a reduction in the uncertainty roughly by a factor between three and four and pushes the growth of the uncertainty bands one decade lower in $x$, which eventually happens when the pseudodata become scarce or have less span in $Q^{2}$.

Similar considerations are in order regarding the impact of DIS pseudodata on the singlet quark helicity distribution
$\Delta \Sigma$, which is shown in Fig. 9. In this case, however, the distribution is already much better constrained by fixedtarget DIS experiments integrated in the DSSV14 analysis. Nevertheless, the impact of the much more precise EIC pseudodata is very significant. Notice that the increased flexibility implemented in the new replica set yield wider bands than those obtained with the DSSV14 set of Ref. [12] at very low- $x$.

For completeness in Fig. 10 we show the helicity distributions $\Delta u+\Delta \bar{u}, \Delta d+\Delta \bar{d}$ and $\Delta s+\Delta \bar{s}$. As in the case of $\Delta \Sigma$, there is a significant improvement compared to the DSSV14 analysis in the region of $x$ where both data and pseudodata overlap $(x>0.001)$. This region combines the precision of EIC pseudodata with the flavor discriminating power of the original DSSV14 dataset. Again, the difference between the two c.m.s. energy configurations is not as relevant as in the case of the gluon helicity because the flavor separation has already been achieved, except at very low- $x$. As usual, the $\Delta u+\Delta \bar{u}$ distribution is much better constrained than those for the other flavors. This feature can be simply traced back to the electric charge factor for $u$ quarks in the proton structure function $g_{1}$, which is four times larger than those for $d$ and $s$ quarks. On the other hand, the latter show rather similar uncertainties at very small- $x$. It is noted that both in DSSV14 and in the variants enhanced with EIC pseudodata we assume $\Delta s=\Delta \bar{s}$.

Rather than the total quark helicities, the quark flavor singlet combination $\Delta \Sigma$, or the gluon helicity distribution $\Delta g$, and their moments, i.e., their integrals over the parton momentum fraction, have a much more simple and intuitive interpretation: the net contribution of quarks and gluons to the proton spin. It is very instructive then to check how well determined are these quantities and how their values and corresponding uncertainty estimates depend on the range of
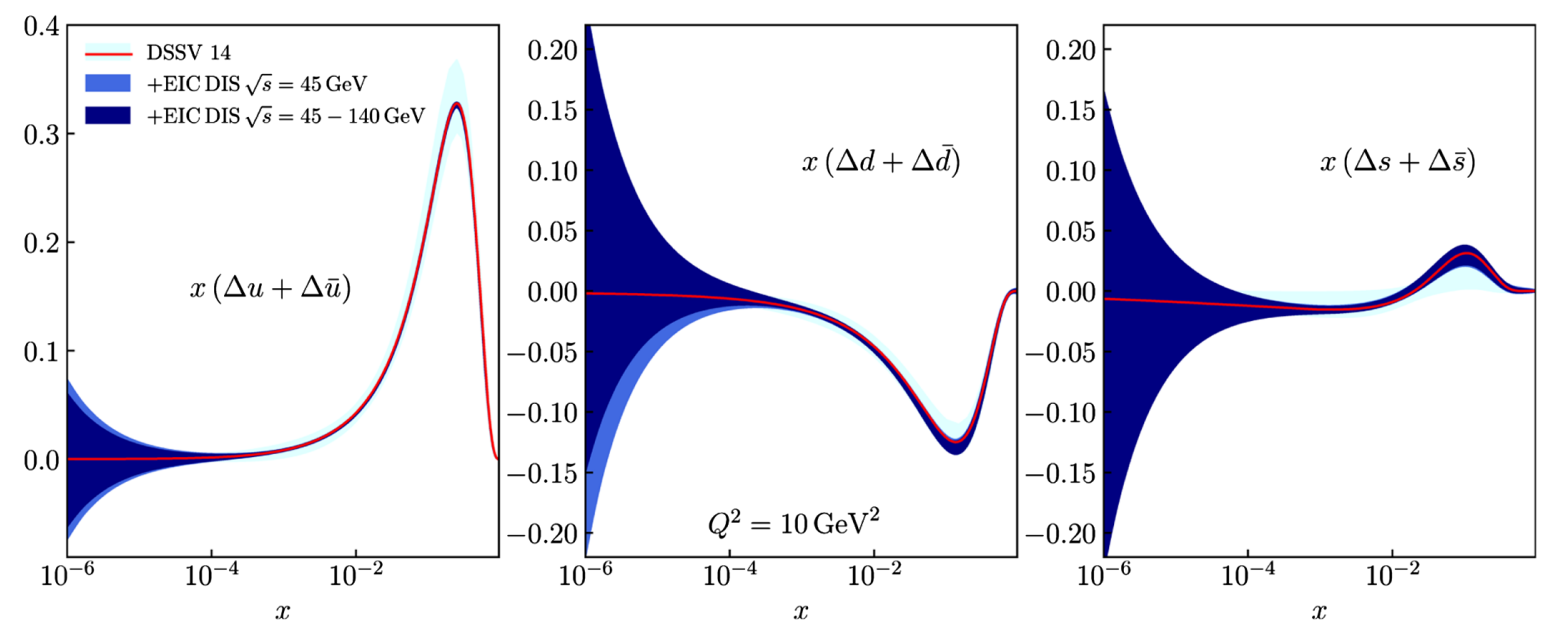

FIG. 10. Impact of the projected EIC DIS data on the total quark helicity distributions $\Delta u+\Delta \bar{d}, \Delta d+\Delta \bar{d}$ and $\Delta s+\Delta \bar{s}$, respectively. Together with the DSSV14 estimate, we show the uncertainty bands resulting from the fit that includes the $\sqrt{s}=44.7$ GeV DIS pseudodata and the reweighting with data at $\sqrt{s}=141.4 \mathrm{GeV}$. 

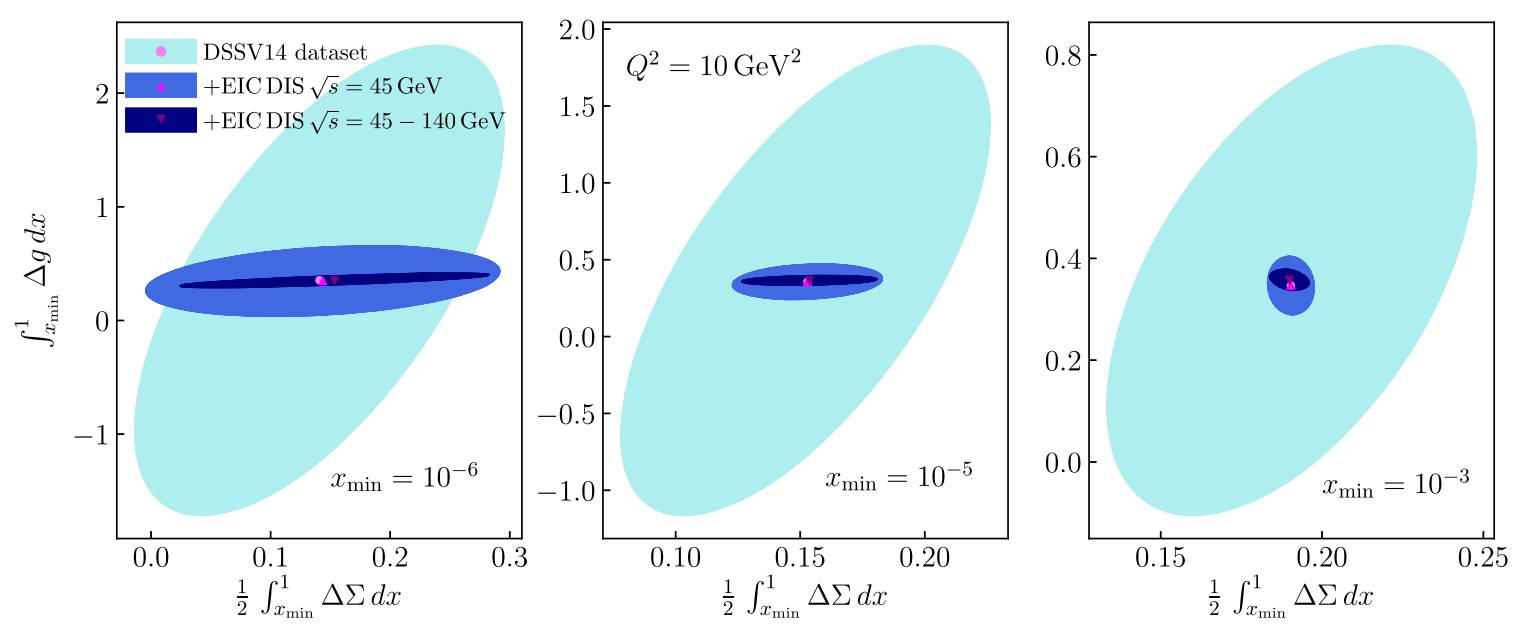

FIG. 11. Correlated uncertainties for the truncated integrals of $\Delta g$ and $\Delta \Sigma$ in the range $10^{-6}<x<1$ (left panel), $10^{-5}<x<1$ (center panel), and $10^{-3}<x<1$ (right panel) computed at $Q^{2}=10 \mathrm{GeV}^{2}$ with DSSV14 (outer area), as well as including pseudodata from the EIC (inner areas).

momentum fraction evaluated. Both quantities are typically correlated so it is natural to represent them in a two dimensional plot, as shown in Fig. 11 for $Q^{2}=10 \mathrm{GeV}^{2}$.

Going from right to left in Fig. 11, the rightmost panel shows the correlated uncertainty of the moments of the gluon (vertical axis) and the quark singlet helicity distributions (horizontal axis) integrated down to $x_{\min }=0.001$, or in other words, the net contribution to the proton spin coming from gluons and quarks down to one thousandth of the proton momentum. The light cyan area represents the range allowed by the data included in the DSSV14 analysis, while the light and darker blue areas denote the impact of the projected EIC measurements. Notice, once again, that the main difference between the impact of both c.m.s. energy configurations is in the constraint on the gluon contribution to the proton spin, roughly by a factor of two.

Clearly, quarks and gluons with smaller momentum fractions may also contribute to the proton spin, and since parton densities tend to be increasingly less constrained towards smaller $x$, the uncertainties on the moments typically grow, as shown in the center and leftmost panels, where the integrals are carried down to $x_{\min }=10^{-5}$ and $x_{\min }=10^{-6}$, respectively. EIC pseudodata stop slightly above $x=10^{-5}$, and below that threshold both the distributions and the moments should be only marginally limited by the continuity, integrability, and positivity of the helicity distributions.

In the DSSV14 low- $x$ extrapolation scenario, the integrals of the singlet quark and gluon helicity distributions saturate rather early, consistent with a picture where at very low- $x$ partons become unpolarized. Therefore, the central values of the truncated moments do not change significantly as lower values are considered for the lower integration limit $x_{\min }$. This feature may or may not be validated by the future EIC measurements, and the chance to verify such behavior emphasizes the relevance of scanning the progression of the moments down to the lowest possible values with the smallest uncertainties.

In Fig. 12, we show again the three area plots of Fig. 11 but now in the same scale, together with the correlated uncertainty of the moments truncated at $x_{\min }=10^{-4}$. The central value of the truncated moments as a function of $x_{\min }$ computed in the DSSV14 extrapolation scenario is plotted as a red curve. A sizable change in the slope of the red curve would suggest that wee partons play a significant role in the proton spin.

Once we have a precise estimate for the constraints that the EIC will impose on the net quark and gluon contribution to the proton spin, it is natural to ask how much room they will leave for other contributions, like the one that could come from quark and gluon orbital angular momentum. For instance, one could assume that the difference

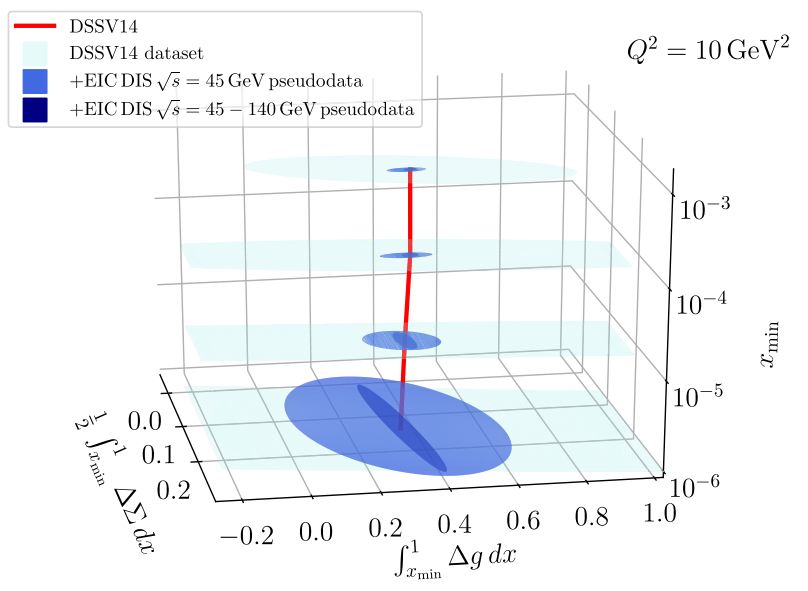

FIG. 12. The same as Fig. 11 but in a common scale for comparison. The red curve represents the central values of the truncated moments as a function of $x_{\min }$, computed extrapolating the DSSV14 scenario. 


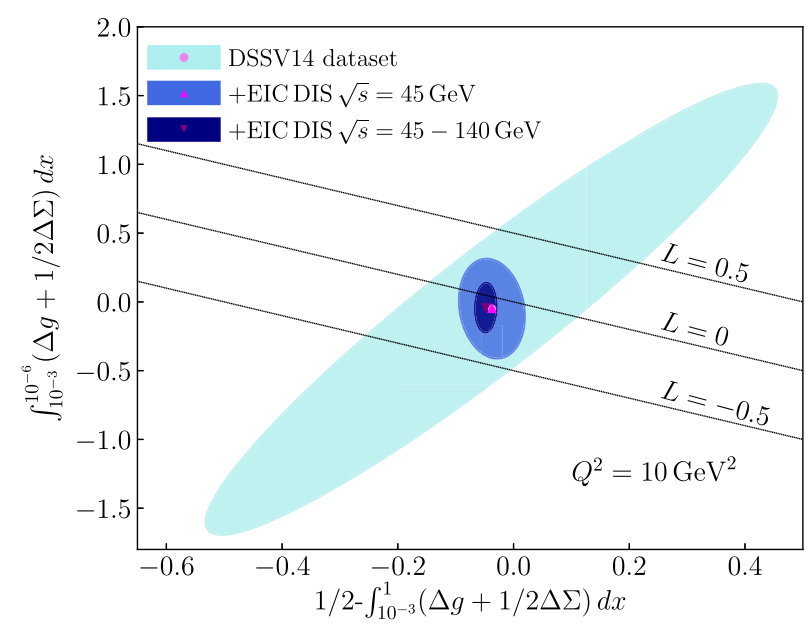

FIG. 13. Room left for potential orbital angular momentum contributions to the proton spin at $Q^{2}=10 \mathrm{GeV}^{2}$, according to present data and future EIC measurements.

between the net quark and gluon spin contribution and the actual proton spin $1 / 2$, could precisely be the contribution from the orbital angular momentum. This is represented in Fig. 13. In the horizontal axis we show the difference between $1 / 2$ and the contribution from the spin of quarks and gluons for a momentum fraction down to $x=0.001$. This would be the room left to the orbital angular momentum if the net spin contribution from partons with smaller momentum fractions is very small or even zero. But as the latter could actually be non-negligible, and is currently very uncertain, we represent in the vertical axis their potential contribution. The colored areas show the constraints on these values coming from present data, in light cyan, and those that one would expect from the projected EIC measurements. The diagonal lines represent the combinations of low and high $x$ contributions for which the resulting orbital angular momentum would be as large as the proton spin and parallel to it, vanishing, or exactly opposite. The EIC data would be able to discard at least one of these extreme scenarios and perhaps, two of them.

\section{B. Impact of semi-inclusive deep inelastic scattering data}

In the following we discuss the impact that the EIC measurements of the semi-inclusive production of charged pions and kaons in collisions between longitudinally polarized electrons and protons will have in constraining helicity of quarks.

We restrict the analysis to transverse-momentum integrated final-state hadrons produced in the currentfragmentation region. Even though the QCD framework to describe transverse-momentum dependent final-state hadron production is known at NLO accuracy [35], as well as hadron production in the target fragmentation region in terms of fracture functions [36,37] in the unpolarized case, the helicity dependent framework is still in development.

As we have already shown in Sec. II B, charged pion and kaon SIDIS spin asymmetries have the potential to pin down sea quark helicities, complementing inclusive DIS measurements, that at least in the electromagnetic case, are unable to disentangle quark and antiquark helicities. Even though the NLO framework for longitudinally polarized DIS processes mediated by weak vector bosons is well known [38], it has not been explored yet, leaving pion and kaon SIDIS as the main tools to probe sea quark polarization as a function of the parton momentum fraction. The EIC allows to extend the kinematical reach of those measurements and improve dramatically their precision.

In Fig. 14 we show the impact of the projected SIDIS measurements on the sea quark helicity distributions. The light cyan bands in the left-hand, center, and right-hand side

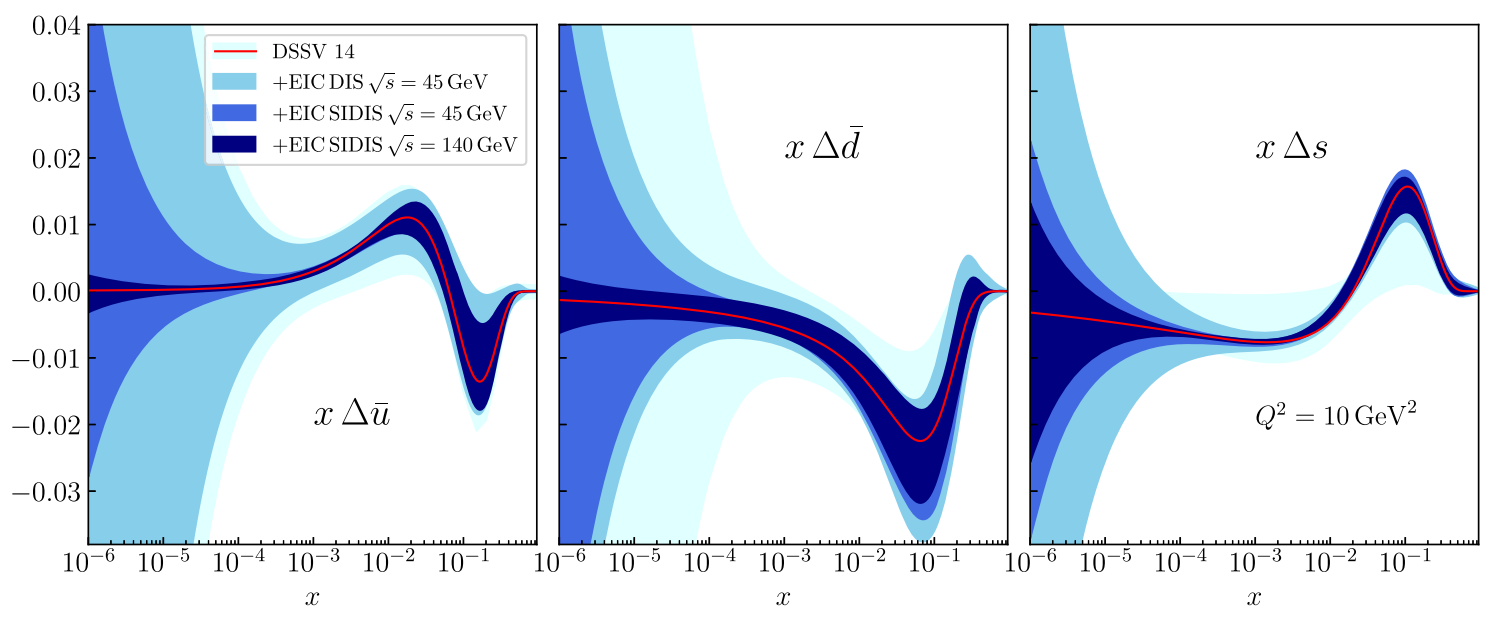

FIG. 14. Impact of the projected EIC SIDIS data on the sea-quark helicity distributions as functions of $x$ at $Q^{2}=10 \mathrm{GeV}$. Together with the DSSV14 estimate, we show the uncertainty bands resulting from the fit that includes the $\sqrt{s}=44.7$ GeV DIS pseudodata and the reweighting with SIDIS pseudodata at $\sqrt{s}=44.7$ and $\sqrt{s}=141.4 \mathrm{GeV}$, respectively. 
panels represent the uncertainty estimates from DSSV14 for $\Delta \bar{u}, \Delta \bar{d}$, and $\Delta \bar{s}$, respectively at $Q^{2}=10 \mathrm{GeV}^{2}$. In the DSSV14 analysis, these distributions are constrained by charged pion, kaon, and unidentified charged hadrons SIDIS data that reach down to $x=5.210^{-3}$. In the case of strange quarks, the charge conjugation symmetry assumption, $\Delta s=\Delta \bar{s}$, together with the hyperon semileptonic $\beta$-decay data on the full moments, constrain further the helicity distribution. The sky blue bands in Fig. 14 show the uncertainty estimated by the Monte Carlo sampling variant of DSSV14 that includes also EIC inclusive DIS pseudodata at $\sqrt{s}=44.7 \mathrm{GeV}$. An inclusive DIS dataset by itself would be unable to constrain the sea-quark densities, however, combined with the SIDIS data already present in the fit, it improve the determination in the region of overlap. This effect is milder for $\Delta \bar{u}$ and $\Delta \bar{d}$, however, in the case of the strange quarks, the impact is much more noticeable, especially at intermediate and large $x$. Remember that the charge conjugation symmetry assumption turns the strange quark distribution effectively into a $\Delta q+\Delta \bar{q}$ quantity for inclusive measurements. On the other hand, the increased flexibility of the new replica set bypasses the hyperon decay constraints at very small- $x$.

The results of reweighting the replicas with EIC SIDIS pseudodata at $\sqrt{s}=44.7$ and $\sqrt{s}=141.4 \mathrm{GeV}$ are shown as light blue and darker blue bands, respectively. The reduction of the uncertainties driven by the inclusion of the SIDIS pseudodata is much more significant for the three sea-quark distributions at $x<0.01$. As usual, $u$ quarks are much better constrained than $d$ quarks because of the charge factor. High c.m.s. energy SIDIS data reach smaller values of the parton momentum fraction, extending the impact on the bands to much smaller values of momentum fraction. For the strange quarks, the constraint combines a more stringent effect due to the charge symmetry assumption with a relatively larger uncertainty in the kaon fragmentation function compared to those for pions, which dilutes the effect of the kaon SIDIS asymmetries.

Given the sharp discriminating power of SIDIS for $\bar{u}$ and $\bar{d}$ quarks, shown in Fig. 14, it would be convenient to release the standard assumption on strange polarization $\Delta s=\Delta \bar{s}$, anticipating that if there is a difference between the polarization of strange quarks and antiquarks, it could be in principle pinned down through the comparison of positively and negatively charged kaon SIDIS measurements at EIC. This is currently beyond the scope of the present exercise but should be implemented in future global analysis, as well as in a quantitative impact assessment.

Another significant advantage of the availability of charge and flavor discriminating SIDIS data is the possibility of performing the full flavor separation without relaying on hyperon semileptonic $\beta$-decay constraints and the flavor symmetry assumptions implicit in their implementation. Global analyses of helicity PDFs routinely use constraints that can be derived from baryonic semileptonic $\beta$-decays under the assumption of $\mathrm{SU}(2)$ and $\mathrm{SU}(3)$ flavor symmetries [39]. These relate combinations of the first moments of the quark helicities to the $F$ and $D$ constants parametrizing the $\beta$-decay rates. However, the use of the beta decay data in this context is controversial; on the one hand because the estimated uncertainties of $F$ and $D$ may not fully reflect the actual breaking of the $\mathrm{SU}(2)$ and, in particular, SU(3) symmetries, for which larger breaking effects have been discussed in the literature [40]. On the other hand, these constraints involve an integration of the helicity distributions over the entire range of momentum fractions, including values of $x$ that cannot be accessed experimentally and for which some kind of extrapolation is necessarily involved.

In the DSSV analyses, rather than imposing the exact $\mathrm{SU}(2)$ and $\mathrm{SU}(3)$ flavor symmetry relations, deviations are allowed and are quantified in terms of two additional free parameters $\varepsilon_{\mathrm{SU}(2)}$ and $\varepsilon_{\mathrm{SU}(3)}$, related to the quark moments and the $F$ and $D$ parameters through

$$
\begin{gathered}
\Delta \Sigma_{u}^{1}-\Delta \Sigma_{d}^{1}=(F+D)\left[1+\varepsilon_{\mathrm{SU}(2)}\right] \\
\Delta \Sigma_{u}^{1}+\Delta \Sigma_{d}^{1}-2 \Delta \Sigma_{s}^{1}=(3 F-D)\left[1+\varepsilon_{\mathrm{SU}(3)}\right],
\end{gathered}
$$

where

$$
\Delta \Sigma_{f}^{1} \equiv \int_{0}^{1}\left[\Delta f_{i}+\Delta \bar{f}_{i}\right]\left(x, \mu_{0}\right) d x .
$$

Note that the free fit parameters $\varepsilon_{\mathrm{SU}(2)}$ and $\varepsilon_{\mathrm{SU}(3)}$ take the place of the normalization of the $\Delta u+\Delta \bar{u}$ and $\Delta d+\Delta \bar{d}$ quark distributions, which otherwise could have been fixed by the measured values of $F$ and $D$. In the analysis, the two combinations including the $F$ and $D$ constants in Eqs. (18) and (19), $F+D$ and $3 F-D$, respectively, are taken as two additional data points, i.e., they are included in the effective $\chi^{2}$ function with their corresponding uncertainties [41] and are treated as any other measurement when performing the minimization. The results for the parameters $\epsilon_{\mathrm{SU}(2)}$ and $\epsilon_{\mathrm{SU}(3)}$ represent the degree of symmetry breaking favored by the data included in the fit, or by the statistically equivalent replicas of the dataset in the MC approach.

In Fig. 15 we show the values obtained for $\varepsilon_{\mathrm{SU}(2)}$ and $\varepsilon_{\mathrm{SU}(3)}$ for the DSSV14 MC replicas (grey dots) and those obtained adding also EIC inclusive DIS proton pseudodata at $\sqrt{s}=44.7$ at $\mathrm{GeV}$ (cyan), respectively. Both replica sets cover a whole range of symmetry breaking values and are mostly concentrated at $\left|\varepsilon_{\mathrm{SU}(2)}\right|<0.01$ and $\left|\varepsilon_{\mathrm{SU}(3)}\right|<0.15$, respectively. As more data with flavor constraining power are included in the analysis, the symmetry breaking parameters should become better determined. This is precisely what happens when EIC SIDIS pseudodata at $\sqrt{s}=44.7$ at $\mathrm{GeV}$ are included through reweighting. In Fig. 15 the green contour represents the $1-\sigma$ limits for the values of $\varepsilon_{\mathrm{SU}(2)}$ and $\varepsilon_{\mathrm{SU}(3)}$ after reweighting with SIDIS 




FIG. 15. Values for the $\varepsilon_{\mathrm{SU}(2)}$ and $\varepsilon_{\mathrm{SU}(3)}$ symmetry breaking parameters defined in Eqs. (18) and (19), respectively, fitted for the DSSV14 MC replicas (grey dots) and those obtained adding also EIC inclusive DIS proton pseudodata at $\sqrt{s}=44.7$ at $\mathrm{GeV}$ (cyan), respectively. The contour lines represent the effect of reweighting with SIDIS pseudodata and inclusive helium measurements in the $1-\sigma$ limits.

pseudodata, to be compared with the blue contour, corresponding to the DSSV14 dataset plus EIC inclusive DIS proton pseudodata at $\sqrt{s}=44.7$ at $\mathrm{GeV}$. The addition of SIDIS data reduces roughly by a factor of 2 the uncertainty on the $\mathrm{SU}(3)$ breaking parameter and also limits sizeably the ones for isospin breaking. Anticipating the results presented in the next section on the EIC He-3 DIS pseudodata, we include in Fig. 15 the $1-\sigma$ limits resulting from the corresponding reweighting as a red contour. As expected, He-3 pseudodata have a very significant effect in probing isospin symmetry but make no further improvement in constraining the $\mathrm{SU}(3)$ symmetry breaking. This highlights the unique importance of SIDIS as a probe of
SU(3) symmetry, through its access to the strange quark helicity, and its complementarity to the inclusive datasets. It also suggests that with the expected level of precision and flavor discrimination, the $\beta$-decay constraints will eventually become not competitive with the combined effect of EIC DIS and SIDIS data.

\section{Impact of deep inelastic scattering off helium}

Even though SIDIS data achieve a remarkable degree of flavor separation, crucially needed for probing sea quarks, the identification of a final-state hadron is always experimentally challenging and, on the other hand, the analysis of such data necessarily involves uncertainties associated to the fragmentation process. In this respect, the availability of polarized light ion beams at the EIC, such as deuterium or helium, would allow to have access to a more direct flavor separation for the combined quark plus antiquark helicity distributions but with the precision characteristic of inclusive measurements. This is especially the case for the very low- $x$ regime, where the flavor separation depends exclusively on the scale dependence when using only proton beams, and for $d$ quarks, in general, since they are typically relegated to a second rank because of both their electric charge factor and their suppression relative to $u$ quarks in the proton.

The impact of the EIC pseudodata on electron-helium collisions at $\sqrt{s}=115.2 \mathrm{GeV}$, estimated by reweighting the DSSV14+EIC45 replicas, is indeed so significant that even though we started with about a thousand replicas, the number of effective replicas that remain with nonnegligible weights is extremely reduced, of order of a dozen replicas, strongly suggesting the need of a new fit. Nevertheless, in the following we show the corresponding results, which must be taken as very rough approximations until a new combined analysis is performed.


FIG. 16. Impact of the projected EIC DIS He data on the total quark helicity distributions $\Delta u+\Delta \bar{d}, \Delta d+\Delta \bar{d}$ and $\Delta s+\Delta \bar{s}$, respectively, as functions of $x$ at $Q^{2}=10 \mathrm{GeV}^{2}$. Together with the DSSV14 estimate, we show the uncertainty bands resulting from the fit that includes the $\sqrt{s}=44.7 \mathrm{GeV}$ DIS pseudodata and the reweighting with He data at $\sqrt{s}=115.2 \mathrm{GeV}$. 


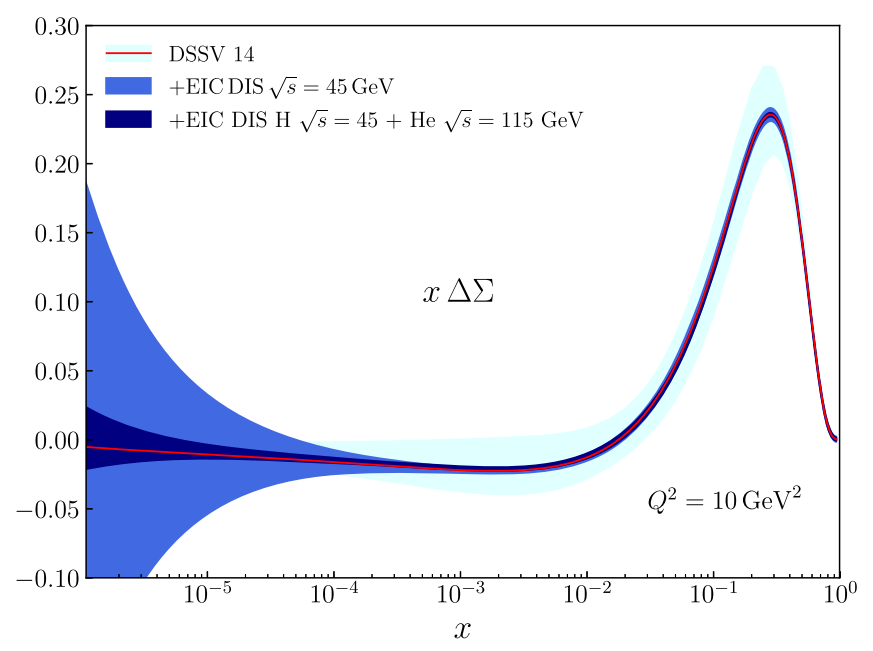

FIG. 17. The same as Fig. 16 but for the singlet quark helicity $\Delta \Sigma$.

In Fig. 16 we show the impact of pseudodata on inclusive electron-helium collisions at $\sqrt{s}=115.2 \mathrm{GeV}$ on the total quark helicity distributions $\Delta u+\Delta \bar{u}, \Delta d+\Delta \bar{d}$ and $\Delta s+\Delta \bar{s}$, respectively. Notice the rather dramatic reduction in the uncertainty bands at very low- $x$ due to the more direct flavor separation. The impact of electron-helium pseudodata on $\Delta \Sigma$ at small $x$ is also quite significant, as can be seen in Fig. 17, where the estimates for $\Delta \Sigma$ are presented in the full range of the parton momentum fraction.

It is worth mentioning that strenuous efforts have been made in the last 25 years to compute the small $x$ asymptotic behavior of helicity distributions within different approaches and formalism, starting with the pioneering work in Refs. [42-45] to the most recent results [46-50]. The extraction of $\Delta \Sigma, \Delta g$ and the individual flavors in the range of momentum fractions $x \sim 10^{-5}-10^{-3}$ would allow to discriminate between the different approaches and approximations and help to determine the onset of these very interesting low- $x$ evolution effects.

The impact from the inclusive DIS electron- $\mathrm{He}-3$ data is also very significant for $\Delta d+\Delta \bar{d}$ and $\Delta \Sigma$ at larger values of momentum fractions, as shown in Fig. 18, where the distributions are normalized to estimates based on the DSSV14 central values. Notice that in the DSSV14 extraction, the sum $\Delta d+\Delta \bar{d}$ is at best constrained at a $10 \%$ level in a very narrow range of $x$ in the valence region, as shown by the light cyan band in the center panel of Fig. 18. The addition of electron-proton EIC pseudodata at $\sqrt{s}=44.7 \mathrm{GeV}$ reduces it to a $5 \%$ level but only in the region where EIC pseudodata overlap with flavor sensitive measurements already present in the DSSV14 dataset. EIC He pseudodata constrains $d$ quark polarization to a $\sim 2 \%$ level down to $x \sim 0.001$, reaching similar precision as for the $u$ quarks, shown in the left panel of Fig. 18. The pseudodata also have a significant impact in the flavor singlet quark helicity, shown normalized to the estimate from the DSSV14 central value in the right panel of Fig. 18. Since the singlet distribution changes sign at $x \sim 0.02$, the ratio is ill defined around that point, but in any case the reduction in the uncertainty is noteworthy, especially at $x>0.15$ where the distribution approaches its maximum.

Finally, the impact on the flavor singlet quark helicity naturally modifies the uncertainty estimate for its truncated moment, which represents the net contribution of the spin of the quark to proton spin. In Sec. IV A we showed that its uncertainty was significantly reduced by electron-proton EIC pseudodata at $\sqrt{s}=44.7 \mathrm{GeV}$, but the addition of $\sqrt{s}=141.4 \mathrm{GeV}$ pseudodata had a mild effect precisely because of the very limited flavor separation. This would not be the case for electron-helium data, as shown in the correlation plot for the truncated moments down to $x=10^{-5}$. The additional pseudodata on electron-helium DIS reduces roughly by more than a factor of 3 the
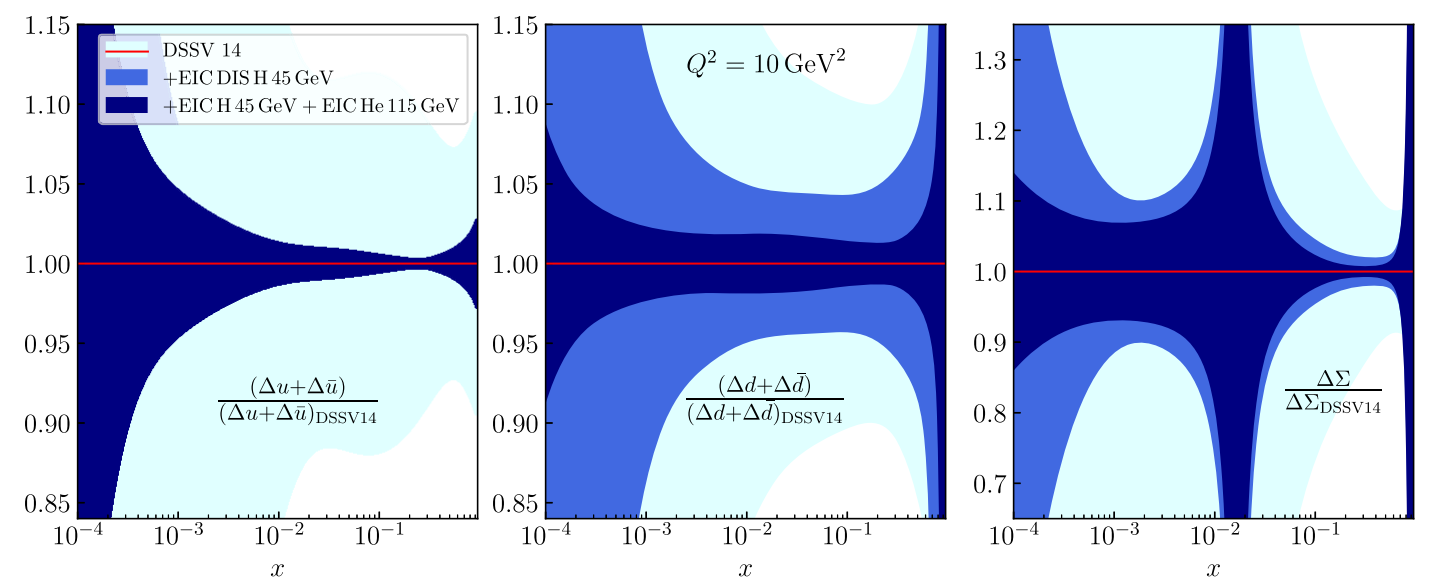

FIG. 18. The same as Fig. 16 but for $\Delta u+\Delta \bar{u}, \Delta d+\Delta \bar{d}$ and $\Delta \Sigma$, respectively, and presented as ratios to the DSSV14 central value in order to show the relative uncertainties of these distributions. 




FIG. 19. Correlated uncertainties for the truncated integrals of $\Delta g$ and $\Delta \Sigma$ in the range $10^{-5}<x<1$ computed at $Q^{2}=$ $10 \mathrm{GeV}^{2}$ with DSSV14 (outer area), as well as including pseudodata from the EIC (inner areas).

uncertainty on the net quark contribution to the proton spin, as shown in Fig. 19.

As it could have been expected, the impact of the electron-helium pseudodata to the gluon contribution to the proton spin is smaller than that of the $\sqrt{s}=141.4 \mathrm{GeV}$ inclusive electron-proton pseudodata mainly because of the more restrictive kinematical coverage of the former. Since the number of surviving replicas obtained by combining the three pseudodatasets in a single reweighting, namely electron-proton DIS at $\sqrt{s}=44.7 \mathrm{GeV}$, $\sqrt{s}=141.4 \mathrm{GeV}$, and electron-helium DIS at $\sqrt{s}=$ $115.2 \mathrm{GeV}$ is extremely low, we defer a detailed discussion of their combined impact to a new global analysis. Presumably, the combined effect would be at least comparable to the intersection of the two darker areas.

\section{SUMMARY}

We have compared the constraining power on the quark and gluon helicity distributions from inclusive and semi-inclusive electron-proton collisions, and inclusive electron-helium collisions, projected to be measured at the future Electron Ion Collider. Combining world data on spin dependent processes included in the DSSV14 analysis with updated estimates for the inclusive electron-proton measurements expected to be obtained in a first stage of the EIC, we built a large set of fit replicas using a Monte Carlo sampling approach and computed the resulting uncertainties. Reweighting the above mentioned replicas with additional pseudodatasets from inclusive electron-proton DIS at a higher c.m.s. energies on semi-inclusive production of pions and kaons, and on inclusive electron-helium collisions, we have assessed and compared the impact on the different helicity distributions.

The overall picture we get is one where the first stage of the EIC with electron-proton collisions at $\sqrt{s}=44.7 \mathrm{GeV}$ reduces the uncertainties of the quark and gluon helicities roughly by a factor of 4 or more down to parton momentum fractions of one thousandth of the proton momentum compared to the DSSV14 estimates. Inclusive data constrain mostly the gluon helicity and the total flavor singlet quark helicity, while semi-inclusive measurements are indispensable to probe the sea quark helicities. A second stage of the EIC with inclusive collisions at $\sqrt{s}=$ 141.4 GeV roughly triples the constraining power on the gluons thanks to the increased range in $x$ and $Q^{2}$, while the semi-inclusive data at this c.m.s. energy improves the sea quark determination down to much smaller values of momentum fractions. Electron-helium collisions, on the other hand, improve the flavor separation for quarks to an unprecedented level, which pushes our reweighting approach to its limits, and suggests the need of a full new fit.

The EIC is designed to bring to conclusion more than forty years of efforts aimed to elucidate the relation between the proton spin and that of its fundamental constituents. This endeavor not only includes the assessment of the role of the gluon spin polarization and the orbital angular momentum of quarks and gluons but more, in general, the relation between spin and flavor, which is at the base of the quark-parton model picture. On the other hand, it also addresses the question about how much of the proton spin depends on those of quarks and gluons carrying negligibly small fractions of the proton momentum. We have investigated to what extent the projected measurements will be able to answer these compelling questions.

\section{ACKNOWLEDGMENTS}

I. B. and R.S. acknowledge Brookhaven National Laboratory for its hospitality and support during the early stages of this work. This work was supported in part by CONICET and ANPCy T. E. C. A. and A. S. N. acknowledge support from the U.S. Department of Energy under Contract No. de-sc0012704. 
[1] J. Ashman et al. (European Muon Collaboration), Nucl. Phys. B328, 1 (1989).

[2] For a review see, e.g., C. A. Aidala, S. D. Bass, D. Hasch, and G. K. Mallot, Rev. Mod. Phys. 85, 655 (2013).

[3] E. C. Aschenauer et al., arXiv:1501.01220.

[4] D. de Florian, R. Sassot, M. Stratmann, and W. Vogelsang, Phys. Rev. Lett. 113, 012001 (2014).

[5] E. R. Nocera et al. (NNPDF Collaboration), Nucl. Phys. B887, 276 (2014).

[6] A. Accardi et al., Eur. Phys. J. A 52, 268 (2016).

[7] E. C. Aschenauer, I. Borsa, R. Sassot, and C. Van Hulse, Phys. Rev. D 99, 094004 (2019).

[8] E. C. Aschenauer, R. Sassot, and M. Stratmann, Phys. Rev. D 86, 054020 (2012).

[9] E. C. Aschenauer, R. Sassot, and M. Stratmann, Phys. Rev. D 92, 094030 (2015).

[10] D. de Florian, R. Sassot, M. Stratmann, and W. Vogelsang, Phys. Rev. Lett. 101, 072001 (2008).

[11] D. de Florian, R. Sassot, M. Stratmann, and W. Vogelsang, Phys. Rev. D 80, 034030 (2009).

[12] D. De Florian, G. A. Lucero, R. Sassot, M. Stratmann, and W. Vogelsang, Phys. Rev. D 100, 114027 (2019).

[13] L. Mankiewicz, A. Schafer, and M. Veltri, Comput. Phys. Commun. 71, 305 (1992).

[14] D. de Florian, R. Sassot, M. Epele, R. J. Hernndez-Pinto, and M. Stratmann, Phys. Rev. D 91, 014035 (2015).

[15] D. de Florian, M. Epele, R. J. Hernandez-Pinto, R. Sassot, and M. Stratmann, Phys. Rev. D 95, 094019 (2017).

[16] A. Kwiatkowski, H. Spiesberger, and H. J. Mohring, Comput. Phys. Commun. 69, 155 (1992); 81, 381 (1994); A. Arbuzov, D. Y. Bardin, J. Blümlein, L. Kalinovskaya, and T. Riemann, Comput. Phys. Commun. 94, 128 (1996).

[17] T. Sjöstrand, S. Ask, J. R. Christiansen, R. Corke, N. Desai, P. Ilten, S. Mrenna, S. Prestel, C. O. Rasmussen, and P. Z. Skands, Comput. Phys. Commun. 191, 159 (2015).

[18] A. Guffanti and J. Rojo, Nuovo Cimento C 033, 65 (2010).

[19] B. T. Wang, T. J. Hobbs, S. Doyle, J. Gao, T. J. Hou, P. M. Nadolsky, and F. I. Olness, Phys. Rev. D 98, 094030 (2018).

[20] L. Del Debbio et al. (NNPDF Collaboration), J. High Energy Phys. 03 (2007) 039.

[21] R. D. Ball et al. (NNPDF Collaboration), Nucl. Phys. B809, 1 (2009); B816, 293(E) (2009).

[22] D. Stump, J. Pumplin, R. Brock, D. Casey, J. Huston, J. Kalk, H. L. Lai, and W. K. Tung, Phys. Rev. D 65, 014012 (2001).

[23] J. Pumplin, D. Stump, R. Brock, D. Casey, J. Huston, J. Kalk, H. L. Lai, and W. K. Tung, Phys. Rev. D 65, 014013 (2001).

[24] R. D. Ball et al. (NNPDF Collaboration), Nucl. Phys. B849, 112 (2011); B854, 926(E) (2012); B855, 927(E) (2012).

[25] R. D. Ball, V. Bertone, F. Cerutti, L. Del Debbio, S. Forte, A. Guffanti, N. P. Hartland, J. I. Latorre, J. Rojo, and M. Ubiali, Nucl. Phys. B855, 608 (2012).

[26] N. Armesto, J. Rojo, C. A. Salgado, and P. Zurita, J. High Energy Phys. 11 (2013) 015.
[27] H. Paukkunen and P. Zurita, J. High Energy Phys. 12 (2014) 100.

[28] I. Borsa, R. Sassot, and M. Stratmann, Phys. Rev. D 96, 094020 (2017).

[29] M. Stratmann and W. Vogelsang, Phys. Rev. D 64, 114007 (2001).

[30] R. Mertig and W. L. van Neerven, Z. Phys. C 70, 637 (1996); W. Vogelsang, Phys. Rev. D 54, 2023 (1996); Nucl. Phys. B475, 47 (1996).

[31] A. Behring, J. Blümlein, A. De Freitas, A. von Manteuffel, and C. Schneider, Nucl. Phys. B897, 612 (2015).

[32] J. Blümlein, G. Falcioni, and A. De Freitas, Nucl. Phys. B910, 568 (2016).

[33] J. Blümlein, C. Raab, and K. Schnwald, Nucl. Phys. B948, 114736 (2019).

[34] J. Ablinger, J. Blümlein, A. De Freitas, A. Goedicke, M. Saragnese, C. Schneider, and K. Schnwald, Nucl. Phys. B955, 115059 (2020).

[35] A. Daleo, D. de Florian, and R. Sassot, Phys. Rev. D 71, 034013 (2005).

[36] A. Daleo and R. Sassot, Nucl. Phys. B673, 357 (2003).

[37] A. Daleo, C. Garcia Canal, and R. Sassot, Nucl. Phys. B662, 334 (2003).

[38] D. de Florian and R. Sassot, Phys. Rev. D 51, 6052 (1995).

[39] For review, see: P. G. Ratcliffe, Czech. J. Phys. 54, B11 (2004).

[40] H. J. Lipkin, Phys. Lett. B 214, 429 (1988); 230, 135 (1989); F. E. Close and R. G. Roberts, Phys. Rev. Lett. 60, 1471 (1988); M. Roos, Phys. Lett. B 246, 179 (1990); Z. Dziembowski and J. Franklin, J. Phys. G 17, 213 (1991); P. G. Ratcliffe, Phys. Lett. B 242, 271 (1990); 365, 383 (1996); arXiv:hep-ph/0012133; S. L. Zhu, G. Sacco, and M. J. Ramsey-Musolf, Phys. Rev. D 66, 034021 (2002); E. Leader and D. B. Stamenov, Phys. Rev. D 67, 037503 (2003).

[41] A. Airapetian et al. (HERMES Collaboration), Phys. Rev. D 75, 012007 (2007).

[42] J. Bartels, B. I. Ermolaev, and M. G. Ryskin, Z. Phys. C 70, 273 (1996).

[43] J. Bartels, B. I. Ermolaev, and M. G. Ryskin, Z. Phys. C 72, 627 (1996).

[44] J. Blümlein and A. Vogt, Phys. Lett. B 370, 149 (1996).

[45] J. Blümlein and A. Vogt, Phys. Lett. B 386, 350 (1996).

[46] Y. V. Kovchegov and Y. Tawabutr, J. High Energy Phys. 08 (2020) 014.

[47] A. Behring, J. Blümlein, A. De Freitas, A. Goedicke, S. Klein, A. von Manteuffel, C. Schneider, and K. Schnwald, Nucl. Phys. B948, 114753 (2019).

[48] Y. V. Kovchegov, D. Pitonyak, and M. D. Sievert, J. High Energy Phys. 01 (2016) 072.

[49] Y. V. Kovchegov, D. Pitonyak, and M. D. Sievert, Phys. Rev. Lett. 118, 052001 (2017).

[50] Y. V. Kovchegov, D. Pitonyak, and M. D. Sievert, Phys. Lett. B 772, 136 (2017). 